The role of actin and myosin in PtK2 spindle length changes induced by laser microbeam irradiations across the spindle

\title{
Rozhan Sheykhani ${ }^{1}$, Norman Baker ${ }^{2 *}$, Veronica Gomez-Godinez ${ }^{3}$, Lih-Huei Liaw ${ }^{4}$, Jagesh Shah $^{5}$, Michael W. Berns ${ }^{6 * *}$, and Arthur Forer ${ }^{1 * *}$
}

${ }^{1}$ Department of Biology, York University, Toronto, Ontario, Canada

${ }^{2}$ Department of Electrical and Computer Engineering, University of California, San Diego,

California

${ }^{3}$ Beckman Laser Institute and Department Developmental and Cell Biology, University of

California, Irvine, California

${ }^{4}$ Beckman Laser Institute, University of California, Irvine, California

${ }^{5}$ Departmnent of Biology, Harvard Medical School, Boston, Massachusetts

${ }^{6}$ Departments of Biomedical Engineering, Developmental and Cell Biology, and Surgery, University of California, Irvine, and Institute for Engineering in Medicine and Department of Bioengineering, University of California, San Diego, California

* The present project is an extension of original observations on spindle shortening described in N.B.'s Ph.D. thesis presented to University of California San Diego in 2010

** Co-corresponding authors

aforer@yorku.ca

(416) 736-5398

mwberns@uci.edu

(858) 822-2954

Running title: Actin and myosin in spindle length control

Keywords: spindle organization, actomyosin, mono-phosphorylated myosin, microtubule ablation, spindle matrix. 


\begin{abstract}
This study investigates spindle biomechanical properties to better understand how spindles function. In this report, laser microbeam cutting across mitotic spindles resulted in movement of spindle poles toward the spindle equator. The pole on the cut side moved first, the other pole moved later, resulting in a shorter but symmetric spindle. Intervening spindle microtubules bent and buckled during the equatorial movement of the poles. Because of this and because there were no detectable microtubules within the ablation zone, other cytoskeletal elements would seem to be involved in the equatorial movement of the poles. One possibility is actin and myosin since pharmacological poisoning of the actin-myosin system altered the equatorial movements of both irradiated and un-irradiated poles. Immunofluorescence microscopy confirmed that actin, myosin and mono-phosphorylated myosin are associated with spindle fibres and showed that some actin and mono-phosphorylated myosin remained in the irradiated regions. Overall, our experiments suggest that actin, myosin and microtubules interact to control spindle length. We suggest that actin and myosin, possibly in conjunction with the spindle matrix, cause the irradiated pole to move toward the equator and that cross-talk between the two half spindles causes the un-irradiated pole to move toward the equator until a balanced length is obtained.
\end{abstract}




\section{Introduction}

Mitotic spindles form de novo at the end of prophase. By metaphase the chromosomes reach the equator and are ready for separation into chromatids that move to opposite poles. As mitotic spindles form the spindle microtubules align along the length of the spindle and assort themselves into non-kinetochore microtubules, kinetochore microtubules, and astral microtubules while dynamically adding and losing subunits and maintaining spindle shape and pole separation. Models that deal with how the spindle is formed and maintained have been reviewed by Goshima and Scholey (2010). Different models emphasize different aspects of how spindles and their microtubules become organised, including: sliding and clustering of spindle microtubules (Burbank et al., 2007); setting up of gradients in Ran-GTP (Wilde and Zheng, 1999; Trieselmann et al., 2003; Clarke, 2005); microtubule depolymerising enzymes at poles and kinetochores (Rogers et al., 2004; Rath and Sharp, 2011); compression forces (Dumont and Mitchison, 2009b); kinesins and other microtubule motor proteins associated with the different kinds of microtubules (Peterman and Scholey, 2009; Civelekoglu-Scholey et el., 2010; Wang et al., 2010); microtubule polymerisation itself, in conjunction with microtubule motors and a tensile matrix (Mitchison et al., 2005); interactions of a spindle matrix with spindle microtubules as the spindle is being formed (Johansen and Johansen, 2007; Johansen et al., 2011) and, relevant to this study, actin and myosin interactions with microtubules (Rosenblatt et al. 2004; Weber et al., 2004; Woolner et al., 2008; Woolner et al., 2009; Rump et al., 2011).

Myosin, actin and microtubules interact with each other in many cytoskeleton systems (Rodriguez et al., 2003). In axons, for example, actin microfilaments and microtubules act against each other to maintain axon length (Solomon and Magendantz, 1988). Tensile forces generated by microfilaments and myosin are antagonistic to the forces generated by cytoplasmic dynein on microtubules: growing axons contract when treated with a microtubule depolymerizing drug, but they do not contract after microtubule depolymerization if the cells are pre-treated with anti-actin or anti-myosin drugs (Ahmad et al., 2000). Since actin and myosin are present in spindles of different organisms (e.g., Xu et a., 2013; reviewed in Forer et al., 2003; Sandquist et al., 2011), actin and myosin might interact in spindles, and there is evidence for this in different species. In crane-fly spermatocytes pharmacological evidence indicates that actin and myosin act with microtubules to move chromosomes and control poleward tubulin flux 
(Silverman-Gavrila and Forer, 2000, Silverman-Gavrila and Forer, 2001; Silverman-Gavrila and Forer, 2003; Fabian and Forer, 2005; Fabian et al., 2007). In Xenopus laevis embryos Myosin 10 and F-actin function antagonistically to maintain spindle length (Woolner et al., 2008). In Dictyostelium discoideum Myosin-1C is necessary to maintain spindle stability and chromosome segregation in association with both spindle microtubules and F-actin (Rump et al., 2011). In PtK (Potorous tridactylus, rat kangaroo) cells, where spindle actin and myosin are seen immunologically (Cande et al., 1977; Sanger et al., 1989; Snyder et al., 2010), different actin and myosin inhibitors cause buckling of microtubules and cause chromosome detachment and blocked chromosome motion (Snyder et al., 2010). In PtK cells, the chemical Y-27632, which inhibits myosin regulatory light chain (MRLC) phosphorylation, interferes with spindle assembly and centrosome positioning (Rosenblatt et al., 2004). Thus these data indicate that actin and myosin might be involved in maintenance of spindle length.

In our experiments, portions of spindles were irradiated. Earlier work using localized irradiation of spindles has given mixed results with respect to its effects on spindle length. Spindle length or shape only rarely was affected in metaphase crane-fly spermatocytes after ultraviolet (UV) microbeam irradiation severed one or several kinetochore spindle fibres (Forer, 1965; Wilson and Forer, 1989), and there was no change in spindle length or shape in Drosophila S2 cells after a laser microbeam severed individual kinetochore fibres (Maiato et al., 2004). There also was no change in length of actin-free spindles assembled from Xenopus egg extracts after microtubules were severed across the spindles (Brugués et al., 2012). Opposite results were obtained in other cells, however. After UV microbeam irradiation severed the microtubules across the entire half-spindle of metaphase newt epithelial cells or PtK2 cells (Snyder et al., 1991), the pole of the irradiated half-spindle moved toward the equator, starting at about the time that microtubules reappeared in the irradiated area (Spurck et al., 1990). After a time delay the poles on the non-irradiated side also moved toward the equator, and the two halfspindles eventually were of the same (shorter) length (Spurck et al., 1990). In other experiments the pole of the irradiated half-spindle immediately moved toward the equatorial plate when individual kinetochore fibres in metaphase newt epithelial cells were irradiated (Forer et al., 1997; Spurck et al., 1997). Thus the two insect cells (Drosophila tissue culture cells and cranefly spermatocytes) respond differently to UV microbeam irradiation than PtK2 or newt epithelial cells, perhaps indicating different organisations in different types of spindles. 
In our experiments a laser microbeam cut across spindles in PtK2 cells stably expressing enhanced cyan fluorescent protein fused to tubulin (ECFP-tubulin). Cutting these spindles causes the irradiated spindle pole to move inward, which usually is followed by the inward movement of the un-irradiated pole. These results raise the question of what produces the force on the pole on the irradiated side in the absence of microtubule connections, and the question of what is the mechanism by which the other pole responds. In this article we investigate the potential roles of actin and myosin in post-ablation reorganization of the spindle. Using immunofluorescence we confirmed the presence of actin and myosin in spindle fibres of PtK2 cells, and pharmacological inhibition of actin polymerization and myosin contraction dramatically altered the inward movements of both the irradiated and un-irradiated poles. The results are relevant to understanding the overall organisation of the mitotic spindle and the role of actin, myosin in maintaining spindle length.

\section{Results}

\section{Laser microbeam irradiation in control cells}

Experiments were done at room temperature and for many of them cells were placed in a fibrin clot, in order to prevent severe rounding up when anti-actin and anti-myosin drugs were added. The same results were obtained when cells were in a clot as when they were not, but the drug-treated cells were of necessity in a clot. To facilitate comparison with experiments done using anti-actin and anti-myosin drugs, the data in this article that act as control irradiations for the drug treatments are presented only from cells in clots. Experiments also were done on cells kept on the microscope stage at $37^{\circ} \mathrm{C}$; we noticed no difference from cells at room temperature except for the slower cell-division transit time at room temperature. Cells under our conditions proceeded through normal anaphase, even after laser irradiations that cut spindles.

Microtubules were severed across the entire widths of 19 metaphase spindles in single line cuts using a second harmonic 532 green picosecond laser. Images of cells were recorded before and after the irradiation. Post-irradiation images were generally recorded every 4 to 8 seconds. Fluorescence disappeared in the irradiated region (Fig. 1A) and the spindle pole on the irradiated side began to move toward the equator from 10-20 seconds after the irradiation (Figs $2 \mathrm{~A}$, and $3 \mathrm{~A}$ ) with a velocity average of $0.7 \mu \mathrm{m} / \mathrm{min}$ (Table 1A). The cut region remained visible 
but filled in within 2-3 minutes (Fig. 1A); our impression from viewing the video sequences is that the gap generally was filled in by movement of the microtubules attached to the pole. After the pole on the irradiated side moved toward the equator, the pole on the opposite side of the spindle began to move toward the equator starting about 50 seconds after the first pole began its motion (Figs 2A and 3A, Table 1A), range: 10s-120s, standard error: 6.9 s. The opposite pole generally moved with the same velocity as the irradiated pole (Table 1A). In 1/19 cells, however, the converse occurred: the pole associated with the cut half-spindle moved toward the equator only after the opposite pole moved. In 3/19 cells only the un-irradiated pole moved; the irradiated one did not (Table 1C). Motion of both poles stopped when the spindle reached an equilibrium length in which the poles were on average around $64 \%$ of their initial distances from the equator (Table 1A). Pre-irradiation spindles were of different lengths in different cells: poleto-pole distances ranged from $9.1 \mu \mathrm{m}$ to $18.9 \mu \mathrm{m}$. Regardless of length, the poles moved inward after line cuts across the spindle (Fig. 3B). In all control cells at least one pole moved toward the equator after line cuts across the spindle (Table 1C). The same general response was obtained independent of whether the cut was made closer to the pole or closer to the equator. Cut spindles that reached reduced length subsequently went through normal anaphase (at that reduced length) so the consequences of cutting the spindles (poles moving toward the equator and shortened spindle) were not responses to lethal damage from the laser. The movements of the poles were not due to changes in cell shape since the cells were held in a fibrin clot and did not change shape after the irradiation (Fig. 1A).

In four cells a single line irradiated across the spindle was repeated in 3 different Zplanes separated by $0.3-0.35 \mu \mathrm{m}$ (Figs 1B, 2B). The two poles responded in the same way as those cut in only a single plane (Table 1B).

To test if spindle microtubules that entered the cut region were responsible for inward motion of the poles, spindles were irradiated with repeated line cuts in a single focal plane in the same position. The first irradiation was in a line across a half spindle. Twenty seconds later, before the half spindle could recover, a repeat irradiation was made at the same position. Repeated irradiations were continued every 20 seconds for 6-9 consecutive irradiations (over 2-3 minutes). In each of the multiple irradiation experiments the spindle poles on the irradiated side immediately moved toward the equator at approximately $0.7 \mu \mathrm{m} / \mathrm{min}$ and reached their final 
positions about $50-60 \%$ of their initial distances from the equator, similar to after single line cuts. The movements of the poles on the non-irradiated sides of the spindles, however, were delayed for the duration of the multiple irradiations. They did not move toward the equator until about 30 seconds after the final irradiation (Fig. 2C). These results suggest that microtubule continuity between pole and equator is not required for the irradiated pole to move toward the equator but that it is required for movement of the opposite pole.

To test if astral microtubules might be responsible for pole movement, we cut astral microtubules. Some cells were irradiated in two lines simultaneously - one on the equatorial side of the pole and the other where the astral microtubules are located between pole and cell membrane. In other cells the pole was enclosed in a box, and the laser beam was scanned over the entire box. After any of these irradiations the poles moved to the equator as described for single line cuts within the spindle indicating that pole movements are not due to pushing by astral microtubules against the cell cortex.

Cuts of spindle regions in the shape of a filled-in rectangular box damaged more microtubules than single line cuts, but the resulting spindle behavior was similar to single line cuts across the spindle. Single line cuts at diagonals (10-45 degrees to the spindle axis) also caused the poles to move, but the poles on the irradiated side often moved at an angle to the spindle axis rather than directly toward the equator and often caused the final shortened spindles to be rotated compared to the original spindle axis. No rotation was seen in line cuts orthogonal to the spindle axis. In the vast majority of cells the pole movements in different irradiation patterns were as described above: the pole associated with the cut half-spindle moved toward the equator first, the other pole later.

When a pole on the non-irradiated side moved toward the equator, in any of the different spindle cuts, the kinetochore spindle fibres between the moving pole and the equator usually became bent, in gradual curves or arcs, or even buckled at very sharp angles (Supplementary movie1, Fig. 1A). Similar bending or buckling was described by Dumont and Mitchison (2009a) after PtK cells were released from compression. When a pole on the irradiated side moved toward the equator, some of the kinetochore fibre stubs that remain attached to the kinetochores or to the poles changed angles after the irradiation, often continuing to change angles as the associated pole moved toward the equator (Figs. 1B). The impression from viewing the video 
sequences is that the poles are not propelled toward the equator by shortening of the microtubules but rather by something external to the microtubules (Supplementary movie 1). That is, the microtubules seem to impede the movement of the pole and the forces from the moving poles cause them either to move laterally or to bend or buckle (Fig. 1A).

It seemed likely that the laser irradiation severed the spindle microtubules, since cytoplasmic microtubules were cut when similar laser irradiation parameters were used (Botvinick et al, 2004). To confirm this for spindle microtubules, irradiated cells were stained with anti-tubulin antibody. We irradiated single lines across spindles in living cells and lysed them very shortly thereafter (for up to 20 minnutes). Once the cells were lysed (but before they were fixed with glutaraldehyde) other spindles were irradiated across the spindle with single lines, double lines or filled-in rectangles in one or multiple focal planes, to determine if the morphology might have been changed by physiological events in vivo that occurred between the time of irradiation and the time of lysis. The spindles irradiated in vivo were identical in all respects to those irradiated after lysis. For cuts in a single plane, the microtubules disappeared in the irradiated focus plane but microtubules remained in other planes (Fig. 4B). In spindles irradiated in 3 different z-planes, however, microtubules were completely absent in all planes (Fig. 4C, 4D). The cell shown in (Fig. 4C), fixed after the near pole started moving toward the equator, has no microtubule continuity across the irradiated region, and thus pole movement takes place without microtubule continuity across the irradiated region. Because poles move toward the equator without microtubule continuity to the equator, and because intervening microtubules bend and buckle, the data suggest that a non-microtubule component causes the poles to move. Actin and myosin might be part of the other component. To test this we studied the effects of actin and myosin inhibitors on pole movement.

\section{Effect of actin inhibitors on equatorial movements of the poles after irradiation}

Actin is present along spindle fibres in PtK cells (Cande et al., 1977; Sanger et al., 1989, Snyder et al., 2010), as confirmed by our phalloidin staining (Fig. 6A). In order to test whether or not actin functions in the inward movement of the irradiated or un-irradiated poles, prior to irradiation cells were treated with either $10 \mu \mathrm{M}$ Cytochalasin D (CD), $2 \mu \mathrm{M}$ Latrunculin B (LatB) or $0.56 \mu \mathrm{M}$ Jasplakinolide (JASP). Both CD and LatB drive F-actin filaments to depolymerise, while JASP stabilises actin filaments (Casella et al., 1981; Morton et al., 2000; Bubb et al., 
2000). To prevent the cells from rounding up after treatment with these drugs (e.g., Snyder and Cohen, 1995; Yvon et al., 2001), the cells were attached to the coverslip using a fibrin clot. Average spindle lengths were shortened by $\mathrm{CD}$ treatment but not by the other actin inhibitors (Fig. 5A). Cytochalasin D caused patches of actin to appear in the cortex and spindle, similar to patches seen after CD treatment described by Tang et al. (1989), while LatB reduced or eliminated phalloidin staining of extra-spindle actin filaments (Fig. 6A). Both CD and LatB caused bending and even buckling of spindle microtubules (Fig. 6A). Some phalloidin staining of spindle fibres remained after treatment with CD and LAT, which was unexpected, but this is similar to results after staining tobacco BY-2 cells: CD removed extra-spindle actin staining and increased the visibility of spindle actin (Yasuda et al., 2005), for reasons considered in detail by them (Yasuda et al., 2005). There was no phalloidin staining visible in jasplakinolide-treated cells because jasplakinolide blocks phalloidin binding to actin (Bubb et al., 1994).

Spindles in treated cells were irradiated in single line cuts after incubating with one of the drugs. All the inhibitors affected the equatorial movement of both poles: in 10/24 cells treated with actin inhibitors there was no movement of either pole whereas in all 19 control cells poles always moved (Table 1C, Fig. 6B), and in some treated cells only the equatorial movement of one pole was affected (Fig. 6C). There was a range of spindle lengths in both control and treated cells and some spindles that were treated were shorter than spindles we studied in the controls. Nonetheless spindle poles moved toward the equator after line cuts in both shorter and longer spindles, so the blocking of pole movements by the inhibitors was not due to inability of small spindles to become shorter (Fig. 5B). Of those cells in which either one or both poles moved toward the equator (Table 1C), the shortening percentage for the entire spindle was about $20 \%$, which is less than the $36 \%$ for control cells (Fig. 5D). For those spindles in which one or both poles moved toward the equator after irradiation of the spindle in the presence of LatB or JASP, both poles moved toward the equator with the same velocities as the corresponding poles of control cells, whereas for the $8 \mathrm{CD}$ treated cells the poles on the irradiated side moved with the same velocities as in control cells but the poles on the un-irradiated side on average moved faster (Fig. 5G).

To test whether the line cuts removed actin in the irradiated region, irradiated control cells were stained for actin. As seen in the confocal microscope, some of the actin extended 
across the irradiated regions, usually localised adjacent to kinetochore-stub or pole-stub microtubules (Fig. 8A).

\section{Effect of myosin inhibitors on equatorial movements of the poles after irradiation}

Both myosin and phosphorylated myosin regulatory light chain (MRLC) are co-localised along spindle microtubules, and both are, as expected, also present throughout the cytoplasm (Figs 7A and B). The myosin and phosphorylated-MRLC staining is punctate along the microtubules, as in other spindles (Fabian et al., 2007). The chromosomes also appear "stained", but this is an inconsistently appearing artefact of our procedures we have not been able to control (for example, confocal images in Fig. 6A for LatB versus Fig. 6A for control and CD). We know it does not represent myosin (or other) staining because we see similar images of chromosomes in channels in which there was no chromophore and even in some preparations that were not stained at all (Figure 7C).

We studied the effects of myosin inhibitors to investigate the role of myosin in the equatorial movements of the spindle poles. The inhibitors were ML-7 $(75 \mu \mathrm{M}), \mathrm{Y}-27632(10 \mu \mathrm{M})$, and BDM (10mM). Blebbistatin could not be used to inhibit myosin because blebbistatin is inactivated by the near UV light used to excite fluorescence in the living cell (Kolega, 2004) and in addition causes loss of fluorescence of the fluorophore (Rosenblatt et al., 2004). Instead we used BDM, since it directly inhibits actin-myosin motility (Forer and Fabian, 2005). The other inhibitors inhibit myosin indirectly by blocking myosin phosphorylation, either by inhibiting Rho-kinase, Y-27632, or by inhibiting myosin light chain kinase, ML-7 (Forer and Fabian 2005, Narumiya et al., 2000 and Saitoh et al., 1987).

The myosin inhibitors had some effects on spindles. Average spindle lengths in ML-7 were shorter than in control cells, but the average lengths in Y-27632 and BDM were not (Fig. 5A). With both ML-7 and Y-27632 the level of 1P-myosin was reduced in the cortex; in some cells treated with myosin inhibitors the astral MTs did not develop and spindle microtubules were bent and buckled (Figs 7A and B), perhaps due to matrix contraction when myosin is inactivated. In some cells chromosomes were detached from the spindle.

Cells treated with myosin inhibitors were irradiated with line cuts across the spindle. All three inhibitors altered the equatorial movements of the poles (Table 1C, Figure 7C and D). 
There was no movement of either pole in 15/31 treated cells whereas poles always moved in control cells (19/19). As with actin inhibitors, some spindle lengths in treated cells were shorter than any in control cells, but since spindle poles moved toward the equator after line cuts in both shorter and longer spindles, the blocking of pole movements by the inhibitors was not due to inability of small spindles to become shorter (Fig. 5C). When in treated cells one or both poles moved toward the equator after line cuts across the spindle, the shortening percentage of the half spindles in the cells in which poles moved after irradiation was less than that in control cells (Fig. 5E). The velocities of the equatorial movements were the same for treated cells and control cells, except for Y-27632 (Fig. 5G), but there were too few cells in which movements occurred (Table 1C) to know if this is a regular phenomenon. When non-treated (control) cells were cut across the spindle and studied using immunofluorescence, punctate 1P-myosin myosin remained in the cut site, but it is not clear whether it was associated with actin filaments or microtubules or both (Fig. 8B).

\section{Discussion}

A variety of laser-mediated spindle cutting geometries applied to PtK cells all resulted in the same phenomenon: a rapid movement of the irradiated pole toward the equator, followed $(\sim 1$ min later) by the un-irradiated pole moving toward the equator with similar speed. Equatorial movements of the poles stopped when the spindles were about $64 \%$ of their initial spindle length. Our data show that microtubules are not present in the cut region, that intervening microtubules bent and buckled, that kinetochore stubs changed angles, and therefore that microtubules are not responsible for the inward movement of either irradiated or un-irradiated poles. On the other hand, the equatorial movement of the poles is perturbed by disruption of actin and myosin.

We first discuss what causes the pole associated with the irradiated half-spindle to move toward the equator.

Forces for the movement of the pole on the irradiated side did not arise from spindle MTs that have remained uncut in the ablation site because there were none: repeated cuts within the spindle did not perturb the pole's motion, nor did cuts in three Z-planes that eliminated all

microtubules throughout the depth of the spindles (Fig. 4C and 4D). Pole movement was not from astral microtubules pushing from the cell cortex because pole movements still occurred 
after cuts around the spindle pole eliminated astral microtubules. Kinetochore microtubules between the moving pole on the un-irradiated side and the equator bent and buckled during pole movements, suggesting that pole motion may arise from compressive forces from source(s) external to the microtubules. Our data suggest that the external sources include actin and myosin.

In cells treated with actin or myosin inhibitors neither pole moved toward the equator in $25 / 55$ cells whereas in control cells one or both poles moved toward the equator in all 19 cells (Table 1C) regardless of initial spindle length (Fig. 3B). In most cells treated with actin or myosin inhibitors the spindle pole on the irradiated side did not move (31/55) whereas in control cells the spindle pole on the irradiated side moved in 16/19 cells (Table 1C), regardless of initial spindle length (Fig. 5B and C). Since actin and myosin (and 1P-myosin), are present in PtK spindles, associated with the MTs (Figs 6A, 7A and B), and some actin and monophosphorylated myosin remained in the irradiated region (Fig. 8A and B), it is reasonable to expect that actin and myosin are at least in part responsible for the equatorial movement of the pole.

Previous experiments also indicated that spindle microtubules respond to nonmicrotubule forces in the spindle. For example, ultraviolet microbeam irradiation of spindles in newt epithelial cells severed several kinetochore fibres across the spindle and caused the shortening of the irradiated half spindle and the bending outwards of the remaining fibres, from which it was concluded that external non-microtubule forces act on spindle fibres and move the irradiated pole inward (Pickett-Heaps et al., 1997). Actin-free spindles formed in Xenopus extracts shortened when treated with a microtubule depolymerising drug and intervening microtubules bent and buckled, from which the authors concluded that extra-microtubule forces caused the reduction of spindle length (Mitchison et al. 2005). After compressing the entire PtK2 cell and causing spindle lengths to increase, release from the compression caused spindle lengths to shorten again; during the shortening the intervening microtubules bent, from which the authors concluded that kinetochore microtubules impede spindle shortening and that spindle shortening arose from "some mechanical element inside or outside the spindle" (Dumont and Mitchison, 2009b). Snyder et al. (2010) similarly found bent microtubules after treatment of PtK cells with actin and myosin inhibitors and suggested that actomyosin is part of a tensile mechanical element along spindle fibres. 
Others also have suggested that actin and myosin are involved in spindle organization and stabilisation. For example, knockdown of unconventional myosin 10 in Xenopus laevis caused mitotic spindle defects and increase in spindle length that were rescued by LatB, which suggested that actin and myosin work antagonistically to maintain spindle length (Woolner et al., 2008). In Dictyostelium discoideum, overexpression of a myosin-1C construct containing a single point mutation in the motor domain caused chromosome misalignment and partially formed spindle fibres (Rump et al., 2011). In fission yeast intact actin is required to stabilize the pre-anaphase mitotic spindle and Latrunculin A delayed the onset of anaphase and disrupted spindle stability (Meadow and Millar, 2008). In crane-fly spermatocytes actin and myosin inhibitors block the elongation of kinetochore stubs formed by ultraviolet microbeam irradiation, also indicating that actin and myosin are involved in spindle function (Forer et al., 2008). Data from Snyder and Cohen (1995) and Snyder et al. (2010) suggest that in PtK cells actin and myosin contribute to mitotic spindle forces and organisation.

Actin and myosin are located in places in the spindle congruent with them playing a role in spindle function. Our immunostaining data demonstrate that actin, myosin and 1P-myosin are localised to spindle fibres (Figs 6A, 7A and B), consistent with previous immunofluorescence microscopy in PtK cells (Cande et al., 1977; Sanger et al., 1989, Snyder et al., 2010) and consistent with the presence of actin and myosin in spindles of other organisms (reviewed by Forer et al., 2003 and Sandquist et al., 2011). Indeed, actin and myosin seem to be associated with spindle microtubules. Though in our experiments this is assessed only by proximity in immunofluorescent images, others have identified similar associations using molecular methods. For example, in Xenopus laevis embryos, unconventional myosin-10 at the poles associates with microtubules by means of a C-terminal MyTH4/FERM domain cassette. It binds to actin via a motor domain in its head and disruption of myosin-10 disrupts spindle assembly (Homma et al., 2001; Weber et al., 2004 and Woolner et al., 2008). In Drosophila melanogaster another MyTH4/FERM superclass myosin (Myosin-15 homolog/Sysiphus) localises to the chromosomes in spindles and interacts with microtubules through microtubule-linked proteins such as Katanin60, EB1, Milton and aPKC (Liu et al., 2008). In Dictyostelium discoideum myosin-1C interacts with microtubules through its tail domain and maintains cross-links between actin filaments and microtubules (Rump et al., 2011). 
Overall, our experiments suggest (a) that actin and myosin are involved in maintaining spindle length and generating forces across the spindle, and (b) that actin and myosin function antagonistically with microtubules. This is consistent with what has been found in axons, where microtubules, actin and myosin interact with each other to maintain axon length, and where tensile forces generated by microfilaments and myosin are antagonistic to the forces generated by microtubules inside the axoplasm (Ahmad et al., 2000).

Two different models could explain the role of actin and myosin in maintaining spindle length. In the first model, similar to suggestions of Woolner et al. (2008) and Rump et al. (2011), myosin localised at the pole and in spindle fibres links to actin by its head domain and to microtubules by its tail domain (Fig. 9A). When the microtubule is cut by laser irradiation the myosin and actin push the irradiated pole toward the equator. While this might explain pole motion, it is does not explain why intervening, impeding microtubules bend or buckle when the poles on the not-irradiated side move in. Rather than work together with microtubules to produce the force, the intervening microtubules seem to resist the force. For that reason, a second preferred model is proposed (Fig. 9B), in which myosin interacts directly with the spindle matrix (Johansen and Johansen, 2007) and with actin, and in which actin filaments and microtubules are linked (Rodriguez et al., 2003). Thus, forces generated by myosin act indirectly on microtubules; when microtubules resist the force moving the poles toward the equator, the intervening microtubules bend or buckle. This model is consistent with the proposed model in which actin, myosin, spindle matrix and microtubules cooperate with each other to generate flux and chromosome movement (Forer et al., 2008) and with our data that remnant actin in the irradiated region is not exactly aligned with spindle microtubules but rather adjacent to microtubules (Fig. 8A). This model is also consistent with the role of spindle matrix in spindle organization, since the matrix is believed to have the requisite elastic properties and could be involved in maintaining spindle length (Johansen and Johansen, 2007; Johansen et al., 2011). It also is consistent with our observations that spindle microtubules sometimes bend when actin and myosin are inhibited (Figs. 6A; 7A, 7B): the matrix might contract when actin/myosin are inhibited. Our interpretation of the laser irradiation and staining results suggests that spindle matrix-related myosin could cooperate with both microtubules and actin filaments in PtK2 cells. 
We now consider why the second pole (on the not irradiated side of the spindle) moves toward the equator some time after irradiation damage to the other half-spindle. Because movement of the second pole is blocked by line cuts repeated every 20 seconds, before recovery of microtubule continuity across the irradiated region can occur, it seems that microtubule continuity is required for starting motion of the second pole. After single line cuts of the cells treated with actin/myosin inhibitors, the equatorial movement of the un-irradiated pole was affected in 35/55 cells (Table 1C). This suggests that non-microtubule components help enable "cross-talk" (feedback) between the two half spindles. This might be related to the "cross-talk" between half spindles in grasshopper spermatocytes uncovered by Nicklas (1979). After differential heating of the two half-spindles and measuring chromosome velocities, Nicklas (1979) concluded that anaphase chromosome velocities were determined by the cooler half spindle, that there is "cross-talk" between the two half-spindles. How might "cross-talk" between half-spindles arise?

Because the equilibrium between tubulin monomers and polymers would be distorted by tubulin depolymerised by the irradiation, one could imagine that the feedback between the two half spindles that leads to balancing of the two sides could be via the tubulin pool. Spurck et al. (1990) argued against cross-talk occurring this way because, if so, both poles would move at the same time. They did not (Spurck et al., 1990), and they did not in the experiments presented herein. How might cross-talk occur, if not via tubulin pools? We suggest that the cross-talk could arise from pole-to-pole tension from actin-myosin-microtubule interactions, or from tension from the spindle matrix. Not only would this tension produce forces to move the irradiated pole toward the equator, it might also maintain the cross-talk between the two half spindles and cause the un-irradiated pole to move inward until a balanced length is obtained between the two halfspindles.

\section{Materials and methods}

\section{Laser and Optical Configuration}

We irradiated spindles using the short-pulsed green second harmonic wavelength of the Nd: YVO4 laser $(532 \mathrm{~nm}$, repetition rate: 76MHz, 12 picoseconds; Vanguard Laser System, Spectra-Physics, Inc., Mountain View, CA) described previously (Botvinick et al., 2004). 
Briefly, the beam was expanded and relayed to the back aperture of the microscope objective $(63 \mathrm{X}, \mathrm{NA}=1.4)$ via the epifluorescence port of an inverted Zeiss microscope (Axiovert 200M, Thornwood, NY). The pulse energy at the focused spot was varied by controlling the orientation of a Glan-Thompson polarizer mounted on a motorized rotational stage (PR50PP, Newport Corporation, Irvine, CA). After passing through the polarizer, the laser beam passed through the microscope to the back aperture of the microscope objective and was then focussed to a near diffraction limited spot. Power in the back aperture of the objective was measured with a power meter/detector (S 120 U.V., Thorlabs, USA). The transmission of the Zeiss Plan-Neofluar 63X/1.4 NA objective was 0.68 , as determined using a multi-objective method previously described (Gomez-Godinez et al., 2010), so the laser pulse energy in the object plane is 0.68 of the measured value. The total exposure time $(30 \mathrm{~ms})$ in the focussed spot, an accumulation of $2.28 \times 10^{6}$ pulses (12 ps each) per focal area of $0.17 \mu \mathrm{m}^{2}$, was controlled by use of a computer controlled mechanical shutter. A scanning pattern of either a line (line-cut) or a filled-in box (box-cut) of numerous $30 \mathrm{~ms}$ exposures was generated by a rapid scanning mirror (FSM-300, Newport Inc., USA), controlled by in-house developed software on a Lab View (National instruments, Houston, TX) platform and National Instrument's data acquisition and control board (Botvinick and Berns, 2005).

For the experiments reported here the laser dosage used was similar to that used previously to cut microtubules (Botvinick et al, 2004). Since the 63X (1.4 NA) microscope objective focuses the beam to a near-diffraction limited spot diameter of approximately $464 \mathrm{~nm}$, the peak irradiance in the laser focal spot is $0.885 \times 10^{-9} \mathrm{~W} / \mathrm{cm}^{2}$. For one $5 \mu \mathrm{m}$ long line scan (with $\sim 10$ laser focal spots/line), $0.43 \mu \mathrm{J}$ of total energy (energy/pulse $\mathrm{x}$ no. of pulses per spot $\mathrm{x}$ no. of spots in the line) was delivered.

\section{Cell Culture}

Potorous tridactylus kidney epithelial cells (PtK2; American Type Culture Collection; \#CCL 56) expressing enhanced cyan fluorescing protein (ECFP) tagged to the $\alpha$-subunit of tubulin as previously described (Botvinick et al., 2004) were grown in Gibco advanced DMEM F-12 supplemented with L-Glutamine and 3\% fetal bovine serum. Cells were seeded into $35 \mathrm{~mm}$ diameter glass bottom (gridded/photo-etched) plastic Petri dishes (MatTek, Ashland, MA) at a density of $3.3 \times 10^{5}$ cells per $\mathrm{ml}$ and allowed to grow for $24-48$ hours at $37^{\circ} \mathrm{C}$ with $5 \% \mathrm{CO}_{2}$ until 
near-confluence at which time they were used for experimentation at a density of approximately $1-1.4 \times 10^{6}$ cells per $\mathrm{ml}$. Some experiments were done with cells directly on the coverslips, but for other experiments cells were adhered to the coverslip before irradiation using a fibrinogenthrombin clot (Forer, 1982; Forer and Pickett-Heaps, 2005; Snyder et al., 2010). The culture dishes were removed from the $37^{\circ} \mathrm{C}$ incubator and placed on the microscope at room temperature $\left(18-20^{\circ} \mathrm{C}\right)$, and mitotic cells were located and irradiated with the laser microbeam.

\section{Software}

The robotic laser microscope software (Robolase) was custom coded for computer control of all hardware and image acquisition in the LabVIEW 8.2 (National Instruments) programming language, as described previously (Botvinick and Berns, 2005). On the livecaptured image of the cell, shapes of either a line-cut $(\sim 5 \mu \mathrm{m})$ or a box-cut ( 2 by $4 \mu \mathrm{m}$ enclosed rectangle) were projected onto the cell image in one or more regions of interest. The Robolase software then calculated the number of pixels inside the designated region and, using the userdefined ablation spot size, calculated the number of $30 \mathrm{~ms}$ exposures necessary to fill in the target. Each $30 \mathrm{~ms}$ exposure received 2,280,000 of 12 ps pulses. The fast scanning mirror (FSM) directed each laser exposure until the entire region of interest was irradiated. A typical spindle irradiation event was completed within 15 seconds.

\section{Camera}

The RoboLase microscope was interfaced with and controlled a Hamamatsu Orca-AG deep-cooled 1,344 X 1,024 pixel 12-bit digital CCD camera (Hamamatsu Photonics, K.K., Hamamatsu, Japan).

\section{Measurements}

For most measurements of length versus time, images were cropped using IrfanView freeware; the resultant individual bmp files were converted into avi files using VirtualDub freeware. Individual images were extracted using WinImage, an in-lab program (Wong and Forer, 2003). Measurements in WinImage were made from a position at the equator, along the spindle axis, mid-way between the poles, chosen so that the two poles were in a straight line that passed through that point. All subsequent measurements were of distances between that fixed 
point and the two poles. For each time point, the position of the equator was entered and each pole was marked with the cursor. After all measurements were made, the program converted inter-pixel distance to $\mu \mathrm{m}$, based on calibration via an image of a stage micrometer, and these were then ported to a commercial program (SlideWrite) to draw graphs and determine speeds. For measurements of lengths in experiments in which there were repeated line cuts at the same position in the spindle, and for box cuts around the aster, individual images were viewed using Image J. In each image lines were drawn from kinetochores at the equator to their pole, and the line lengths in pixels were recorded. After all images in a sequence were measured the inter-pixel distances were converted to $\mu \mathrm{m}$ based on the inter-pixel distances in images of a stage micrometer. Lengths for each time point were recorded and put into Excel to generate graphs of distance versus time.

\section{Drug treatment}

Seeded PtK2 cells (as above) were adhered to the coverslip with a fibrinogen-thrombin clot and then treated and incubated at $37^{\circ} \mathrm{C}$ for 15 to 20 minutes with one of the following drugs: $10 \mu \mathrm{M}$ Cytochalasin D (CD) (Tocris Bioscience, Bristol, UK), $2 \mu \mathrm{M}$ Latrunculin B (LatB) (Calbiochem, Billerica, MA, USA), $0.56 \mu \mathrm{M}$ Jasplakinolide (JASP) (Calbiochem, Billerica, MA, USA), $10 \mathrm{mM}$ 2,3-Butanedione monoxime (BDM) (Sigma, Burlington, ON, Canada), $10 \mu \mathrm{M}$ Y27632 (trans-4-[(1R)-1-Aminoethyl]-N-4-pyridinylcyclohexanecarboxamide dihydrochloride) (Tocris Bioscience, Bristol, UK) or $75 \mu \mathrm{M}$ Hexahydro-1-[(5-iodo-1-naphthalenyl)sulfonyl]-1 $\mathrm{H}$ 1,4-diazepine hydrochloride (ML-7) (Toronto Research Chemicals, Toronto, ON, Canada). The inhibitors were dissolved in DMSO or medium at 1000x the final concentration, then diluted into medium and added to the cells in the clot. The cells were studied at room temperature in the presence of the drug.

\section{Fluorescent staining and confocal microscopy}

We used the fixation-staining protocol described by Fabian and Forer (2005). After treatment and/or laser irradiation of spindle fibres, PtK2 cells were lysed for up to 20 minutes at room temperature in a large volume $(>5 \mathrm{ml})$ of lysis buffer $(100 \mathrm{mM}$ piperazineN,N-bis(2ethanesulfonic acid) [PIPES]; 10 mM EGTA; 5 mM MgSO4; 5\% DMSO; 1\% Nonidet P-40; pH 6.9), and then rinsed in lysis buffer. Lysis was probably within 10 seconds (as quickly as we 
could add the lysis medium to the cells after the irradiation). In some cells we delayed addition of lysis buffer for 25-45 seconds, to allow time for the near pole to move toward the equator. After irradiation of lysed cells on the same coverslips, the cells were fixed for 6 minutes in $0.25 \%$ glutaraldehyde in phosphate-buffered saline (PBS), rinsed in PBS (two times for 5 minutes each), kept for 10 to 15 minutes in sodium borohydride $(1 \mathrm{mg} / \mathrm{ml})$ to neutralise free aldehyde groups, rinsed again with PBS (two times for 5 minutes each) and stored in PBSglycerol 1:1 (v/v) at $4^{\circ} \mathrm{C}$. After rinsing away the glycerol, irradiated or treated cells were double stained with actin-tubulin, myosin-tubulin or mono-phosphorylated myosin (regulatory light chain 1P-myosin)-tubulin. All staining steps were done at room temperature in MatTek glass bottom dishes. Glycerol:PBS was removed prior to staining by rinsing the dishes with PBS (two times for 20 minutes each); they were rinsed with PBS containing $0.1 \%$ Triton X-100 before adding antibody or phalloidin, to facilitate spreading of the antibody (or phalloidin) on the coverslip. The incubation time for each antibody and for phalloidin was 1 hour; after each incubation period, the cells were rinsed two times for 5 min each with PBS and then with PBS containing $0.1 \%$ Triton X-100. Preparations were kept in the dark during the incubation periods to prevent light inactivation of the fluorochromes.

For actin-tubulin staining, actin filaments were stained with $0.66 \mu \mathrm{M}$ Alexa 568 phalloidin (Invitrogen, Burlington, ON, Canada); tubulin was stained with YL1/2 rat monoclonal antibody specific for tyrosinated $\alpha$-tubulin (Kilmartin et al., 1982) diluted (1:200), followed by Alexa 633 goat anti-rat immunoglobulin IgG (Invitrogen, Burlington, ON, Canada) diluted 1:100. For myosin-tubulin staining, myosin was stained with MY-21, a mouse IgM antibody against myosin regulatory light chain (Sigma), diluted 1:100, followed by Alexa 488 Goat antimouse IgM (Invitrogen, Burlington, ON, Canada), diluted 1:100. Tubulin was stained with YL1/2 rat monoclonal antibody specific for tyrosinated $\alpha$-tubulin diluted (1:200), followed by Alexa 594 goat anti-rat immunoglobulin IgG (Invitrogen, Burlington, ON, Canada) diluted 1:100. For $1 \mathrm{P}$ myosin-tubulin staining, mono phosphorylated myosin regulatory light chain was stained with Phospho-Myosin Light Chain 2 (Ser19) Mouse IgG antibody (Cell Signaling (NEB), Pickering, ON, Canada) diluted 1:150 followed by Alexa 488 Goat anti-mouse IgG, diluted 1:200. Tubulin was stained as in myosin-tubulin preparations. All dilution of antibodies was done in PBS. After staining and before mounting, the glass coverslip was removed from the dish using coverslip removal fluid (MatTek, Ashland, MA, USA). Coverslips were mounted in 
Mowiol (Calbiochem, Billerica, MA, USA) solution (Osborn and Weber, 1982) containing paraphenylene diamine (PPD) as antifading agent (Fabian and Forer, 2005), and stored at $4^{\circ} \mathrm{C}$ in the dark.

Cells were studies using an Olympus Fluoview 300 confocal microscope, with argon laser at $488 \mathrm{~nm}$ and $\mathrm{HeNe}$ laser at $543 \mathrm{~nm}$ and $660 \mathrm{~nm}$, using an Olympus Plan Apo 60X oil immersion objective (numerical aperture, 1.4). Images, collected with Fluoview software, were further processed using Image $\mathrm{J}$ and Adobe Photoshop.

\section{Acknowledgements}

We would like to thank Dr. Michelle Duquette-Huber (University of California, San Diego) for her help in preparing the cells and reagents. We are grateful to Dr. Linda Shi (University of California, San Diego) for her help and guidance with the Robolase microscope. This work was supported by grants from the Canadian Natural Sciences and Engineering Research Council to A.F., and by grants from Beckman Laser Institute Foundation and the Air Force Office of Scientific Research (AFOSR \# F9620-00-1-0371) to M.W.B. 


\section{References}

Ahmad FJ, Hughey J, Wittmann T, Hyman A, Greaser M, and Baas, PW. 2000. Motor proteins regulate force interactions between microtubules and microfilaments in the axon. Nat Cell Biol. 2: $276-80$.

Botvinick EL, Venugopalan V, Shah JV, Liaw LH, and Berns MW. 2004. Controlled ablation of microtubules using a picosecond laser. Biophys J. 87:4203-12.

Botvinick EL, and Berns MW. 2005. Internet-based robotic laser scissors and tweezers microscopy. Microsc. Res. Tech. 68:65-74.

Brugués J, Nuzzo V, Mazur E, and Needleman DJ. 2012. Nucleation and transport organize microtubules in metaphase spindles. Cell. 149: 554-64.

Bubb MR, Lewis MS, and Korn ED. 1994. Actobindin binds with high affinity to a covalently cross-linked actin dimer. J Biol Chem. 269: 25587-91.

Bubb MR, Spector I, Beyer BB, and Fosen KM. 2000. Effects of jasplakinolide on the kinetics of actin polymerization An explanation for certain in vivo observations. J Biol Chem. 275: 5163-70.

Burbank KS, Mitchison TJ, and Fisher DS. 2007. Slide-and-cluster models for spindle assembly. Curr Biol. 17: 1373-83.

Cande WZ, Lazarides E, and McIntosh JR. 1977. A comparison of the distribution of actin and tubulin in the mammalian mitotic spindle as seen by indirect immunofluorescence. J Cell Biol. 72: $552-567$.

Civelekoglu-Scholey G, Tao L, Brust-Mascher I, Wollman R, and Scholey JM. 2010. Prometaphase spindle maintenance by an antagonistic motor-dependent force balance made robust by a disassembling lamin-B envelope. J Cell Biol. 188: 49-68.

Clarke PR. 2005. A gradient signal orchestrates the mitotic spindle. Science. 309: 1334-5. 
Casella JF, Flanagan MD, and Lin S. 1981. Cytochalasin D inhibits actin polymerization and induces depolymerization of actin filaments formed during platelet shape change. Nature. 293: 302-305.

Dumont S, and Mitchison TJ. 2009a. Force and length in the mitotic spindle. Curr Biol. 19: R749-61.

Dumont S, and Mitchison TJ. 2009b. Compression regulates mitotic spindle length by a mechanochemical switch at the poles. Curr Biol. 19: 1086-95.

Fabian L, and Forer A. 2005. Redundant mechanisms for anaphase chromosome movements: crane-fly spermatocyte spindles normally use actin filaments but also can function without them. Protoplasma. 225: 169-84.

Fabian L, Troscianczuk J, Forer A. 2007. Calyculin A, an enhancer of myosin, speeds up anaphase chromosome movement. Cell Chromosome. 6:1.

Forer A. 1965. Local reduction of spindle fibre birefringence in living Nephrotoma Suturalis (Loew) spermatocytes induced by ultraviolet microbeam irradiation. J Cell Biol. 25: 95-117.

Forer A. 1982. Crane-fly spermatocytes and spermatids: a system for studying cytoskeletal components. Methods Cell Biol. 25: 227-52.

Forer A, Spurck T, and Pickett-Heaps JD. 1997. Ultraviolet microbeam irradiations of spindle fibres in crane-fly spermatocytes and newt epithelial cells: resolution of previously conflicting observations. Protoplasma. 197: 230-40.

Forer A, Spurck T, Pickett-Heaps JD, and Wilson PJ. 2003. Structure of kinetochore fibres in crane-fly spermatocytes after irradiation with an ultraviolet microbeam: neither microtubules nor actin filaments remain in the irradiated region. Cell Motil Cytoskeleton. 56: 173-92.

Forer A, and Fabian L. 2005. Does 2,3-butanedione monoxime inhibit nonmuscle myosin?. Protoplasma. 225: 1-4.

Forer A, and Pickett-Heaps JD. 2005. Fibrin clots keep non-adhering living cells in place on glass for perfusion or fixation. Cell Biol Int. 29: 721-30. 
Forer A, Pickett-Heaps JD, and Spurck T. 2008. What generates flux of tubulin in kinetochore microtubules? Protoplasma 232: 137-41.

Gomez-Godinez V, Wu T, Sherman AJ, Lee CS, Liaw LH, Zhongsheng Y, Yokomori K, and Berns MW. 2010. Analysis of DNA double-strand break response and chromatin structure in mitosis using laser microirradiation. Nucleic Acids Res. 38: 1-18.

Goshima G, and Scholey JM. 2010. Control of mitotic spindle length. Annu Rev Cell Dev Biol. 26: 21-57.

Homma K, Saito J, Ikebe R, and Ikebe M. 2001. Motor function and regulation of myosin X. J Biol Chem. 276: 34348-54.

Johansen KM, and Johansen J. 2007.Cell and molecular biology of the spindle matrix. Int Rev Cytol. 263: 155-206.

Johansen KM, Forer A, Yao C, Girton J, and Johansen J. 2011. Do nuclear envelope and intranuclear proteins reorganize during mitosis to form an elastic, hydrogel-like spindle matrix? Chromosome Res. 3: 345-65.

Kilmartin JV, Wright B, and Milstein C. 1982. Rat monoclonal antitubulin antibodies derived by using a new nonsecreting rat cell line. J Cell Biol. 93: 576-82.

Kolega J. 2004. Phototoxicity and photoinactivation of blebbistatin in UV and visible light. Biochem Biophys Res Commun. 320: 1020-5.

Liu R, Woolner S, Johndrow JE, Metzger D, Flores A, and Parkhurst SM. 2008. Sisyphus, the Drosophila myosin XV homolog, traffics within filopodia transporting key sensory and adhesion cargos. Development 135: 53-63.

Maiato H, Rieder CL, and Khodjakov A. 2004. Kinetochore-driven formation of kinetochore fibers contributes to spindle assembly during animal mitosis. J Cell Biol. 167: 831-40.

Meadows JC, and Millar J. 2008. Latrunculin A delays anaphase onset in fission yeast by disrupting an Ase1-independent pathway controlling mitotic spindle stability. Mol Biol Cell. 19: 3713-23. 
Mitchison TJ, Maddox P, Gaetz J, Groen A, Shirasu M, Desai A, Salmon ED, and Kapoor TM. 2005. Roles of polymerization dynamics, opposed motors, and a tensile element in governing the length of Xenopus extract meiotic spindles. Molec. Boil. Cell. 16: 3064-76.

Morton WM, Ayscough KR, and McLaughlin PJ. 2000. Latrunculin alters the actin-monomer subunit interface to prevent polymerization. Nat Cell Biol. 2: 376-8.

Narumiya S, Ishizaki T, and Uehata M. 2000. Use and properties of ROCK-specific inhibitor Y27632. Methods Enzymol. 325: 273-84.

Nicklas RB. 1979. Chromosome movement and spindle birefringence in locally heated cells: interaction versus local control. Chromosoma 74: 1-37.

Osborn M, and Weber K. 1982. Immunofluorescence and immunocytochemical procedures with affinity purified antibodies: tubulin-containing structures. Methods Cell Biol. 24: 97-132.

Peterman EJ, and Scholey JM. 2009. Mitotic microtubule crosslinkers: insights from mechanistic studies. Curr Biol. 19: R1089-94.

Pickett-Heaps JD, Forer A, and Spurck T. 1997. Traction fibre: toward a "tensegral" model of the spindle. Cell Motil Cytoskeleton 37: 1-6.

Rath U, and Sharp DJ. 2011. The molecular basis of anaphase A in animal cells. Chromosome Res. 19: 423-32.

Rodriguez OC, Schaefer AW, Mandato CA, Forscher P, Bement WM and Waterman-Storer CM. 2003. Conserved microtubule-actin interactions in cell movement and morphogenesis. Nat Cell Biol. 7: 599-609.

Rogers GC, Rogers SL, Schwimmer TA, Ems-McClung SC, Walczak CE, Vale RD, Scholey JM, and Sharp DJ. 2004. Two mitotic kinesins cooperate to drive sister chromatid separation during anaphase. Nature 427: 364-70.

Rosenblatt J, Cramer LP, Baum B, and McGee KM. 2004. Myosin II-dependent cortical movement is required for centrosome separation and positioning during mitotic spindle assembly. Cell. 117, 361-72. 
Rump A, Scholz T, Thiel C, Hartmann FK, Uta P, Hinrichs MH, Taft MH, and Tsiavaliaris G. 2011. Myosin-1C associates with microtubules and stabilizes the mitotic spindle during cell division. J Cell Sci. 124: 2521-8.

Saitoh M, Ishikawa T, Matsushima S, Naka M, and Hidaka H. 1987. Selective inhibition of catalytic activity of smooth muscle myosin light chain kinase. J Biol Chem. 262: 7796-801.

Sandquist JC, Kita AM, and Bement WM. 2011. And the dead shall rise: actin and myosin return to the spindle. Dev Cell. 21: 410-419.

Sanger JM, Mittal B, Dome JS, and Sanger JW. 1989. Analysis of cell division using fluorescently labeled actin and myosin in living PtK2 cells. Cell Motil Cytoskeleton 14: 201-19.

Silverman-Gavrila RV, and Forer A. 2000. Evidence that actin and myosin are involved in the poleward flux of tubulin in metaphase kinetochore microtubules of crane-fly spermatocytes. J. Cell Sci. 113: 597-609.

Silverman-Gavrila RV, and Forer A. 2001. Effects of anti-myosin drugs on anaphase chromosome movement and cytokinesis in crane-fly primary spermatocytes. Cell Motil Cytoskeleton 50: 180-97.

Silverman-Gavrila RV, and Forer A. 2003. Myosin localization during meiosis I of crane-fly spermatocytes gives indications about its role in division. Cell Motil Cytoskeleton 55: 97-113.

Snyder JA, Armstrong L, Stonington OG, Spurck TP, and Pickett-Heaps JD. 1991. UVmicrobeam irradiations of the mitotic spindle: spindle forces and structural analysis of lesions. Eur J Cell Biol. 55: 122-32.

Snyder JA, and Cohen L. 1995. Cytochalasin J affects chromosome congression and spindle microtubule organization in PtK1 cells. Cell Motil Cytoskeleton 32: 245-57.

Snyder JA, Ha Y, Olsofka C, and Wahdan R. 2010. Both actin and myosin inhibitors affect spindle architecture in PtK1 cells: does an actomyosin system contribute to mitotic spindle forces 
by regulating attachment and movements of chromosomes in mammalian cells? Protoplasma 240: 57-68.

Solomon F, and Magendantz M. 1988. Cytochalasin separates microtubule disassembly from loss of asymmetric morphology. J. Cell Biol. 89: 157-61.

Spurck TP, Stonington OG, Snyder JA, Pickett-Heaps JD, Bajer A, and Mole-Bajer J. 1990. UV microbeam irradiations of the mitotic spindle. II. Spindle fiber dynamics and force production. J. Cell Biol. 111: 1505-18.

Spurck T, Forer A, and Pickett-Heaps J. 1997. Ultraviolet microbeam irradiations of epithelial and spermatocyte spindles suggest that forces act on the kinetochore fibre and are not generated by its disassembly. Cell Motil Cytoskeleton 36: 136-48.

Tang, XJ, Lancelle SA, and Hepler PK. 1989. Fluorescence microscopic localization of actin in pollen tubes: comparison of actin antibody and phalloidin staining. Cell Motil Cytoskeleton 12: 216-24.

Trieselmann N, Armstrong S, Rauw J, and Wilde, A. 2003. Ran modulates spindle assembly by regulating a subset of TPX2 and Kid activities including Aurora A activation. J Cell Sci. 116: 4791-98.

Wang H, Brust-Mascher I, Cheerambathur D, and Scholey JM. 2010. Coupling between microtubule sliding, plus-end growth and spindle length revealed by kinesin-8 depletion. Cytoskeleton (Hoboken) 11: 715-28.

Weber KL, Sokac AM, Berg JS, Cheney RE, and Bement WM. 2004. A microtubule-binding myosin required for nuclear anchoring and spindle assembly. Nature 431: 325-429.

Wilde A, and Zheng Y. 1999. Stimulation of microtubule aster formation and spindle assembly by the small GTPase Ran. Science 284: 1359-62.

Wilson P, and Forer A. 1989. The behaviour of microtubules in chromosomal spindle fibres irradiated singly or doubly with ultraviolet light. J Cell Sci. 94: 625-34. 
Wong R, Forer A. 2003. "Signalling” between chromosomes in crane-fly spermatocytes studies using ultraviolet microbeam irradiation. Chromosome Res. 11: 771-86.

Woolner S, O'Brien LL, Wiese C, and Bement WM. 2008. Myosin-10 and actin filaments are essential for mitotic spindle function. J Cell Biol. 182: 77-88.

Woolner S, and Bement WM. 2009. Unconventional myosins acting unconventionally. Trends Cell Biol. 19: 245-52.

Xu C, Liu Z, Zhang L, Zhao C, Yuan S, and Zhang F. 2013. Organization of actin cytoskeleton during meiosis I in a wheat thermo-sensitive genic male sterile line. Protoplasma 250:415-422.

Yasuda H. Kanda K, Koiwa H, Suenaga K, Kidou S, and Ejiri S. 2005. Localization of actin filaments on mitotic apparatus in tobacco BY-2 cells. Planta. 222: 118-29.

Yvon, A M-C, Gross, D J, and Wadsworth, P. 2001. Antagonistic forces generated by myosin II and cytoplasmic dynein regulate microtubule turnover, movement, and organization in interphase cells. Proc Natl Acad Sci USA 98: 8656-8661 


\section{Figure legends:}

Figure 1 (A) Montage of images of a cell before and after single line irradiation across the spindle. The loss of fluorescence in the cut region is indicated by an arrow in the image at 10:38:16. The arrowhead at 10:38:16, 10:38:33, 10:38:48, and 10:40:07 points to a kinetochore microtubule bundle that bent as the pole on the un-irradiated side of the spindle moved toward the equator. The bend is more severe at later times. The shape of the cells did not change after the irradiation. Bar: $10 \mu \mathrm{m}$. (B) Montage of images of a cell before and after single line irradiation in multiple focal planes across the spindle. The loss of fluorescence in the cut region is indicated by an arrow in the image at 12:58:11. The arrowhead points to the kinetochore fibre stub that remained attached to the kinetochores but that changed angles after the irradiation. The fluorescence often is photobleached during observation using fluorescence microscopy and the images in this cell show the fading that can occur. Immunofluorescence images (described later) indicate that this is fading, and not loss of microtubules per se. Bar: $10 \mu \mathrm{m}$.

Figure 2 (A) Graph of distance of the irradiated and un-irradiated poles from the equator in $\mu \mathrm{m}$, versus time in minutes for the cell illustrated in Fig. 1A. The vertical line represents the time of the irradiation. The pole on the irradiated side moved toward the equator first, shortly after the irradiation, with a velocity of $0.94 \mu \mathrm{m} / \mathrm{min}$. (B) Graph of distance of the irradiated and unirradiated poles from the equator in $\mu \mathrm{m}$, versus time in minutes for the cell illustrated in Fig. 1B. The pole on the irradiated side moved toward the equator first, shortly after the irradiation, with a velocity of $1.4 \mu \mathrm{m} / \mathrm{min}$. (C) Graph of distance from the equator, in $\mu \mathrm{m}$, versus time, in minutes, for a cell irradiated in the same line repeatedly, at 20 second intervals. The horizontal line in the middle represents the duration of the repeated irradiations. The pole on the irradiated side moved toward the equator starting shortly after the first cut with velocity of $1 \mu \mathrm{m} / \mathrm{min}$, until it reached its equilibrium position. The other pole did not start to move toward the equator until after the last cut. It moved with velocity of $0.8 \mu \mathrm{m} / \mathrm{min}$ until it reached its equilibrium position. For all the graphs the (blue) crosses represent the positions of the pole on the irradiated side of the spindle and the (red) triangles represent the positions of the other pole. The dashed lines are lines of best fit to the points indicated by tiny symbols at the centers of the marked crosses and the horizontal 
line at the bottom represents the delay of movement for un-irradiated pole to move in after irradiation.

Figure 3 (A) Time range required (in seconds) for irradiated and un-irradiated pole to start to move towards the equator after laser microbeam irradiation. (B) The consequences of line cuts in spindles of different lengths, showing the spindle length distribution in control cells before laser microbeam irradiation.

Figure 4 Un-irradiated (A) and irradiated (B, C and D) PtK2 cells stained with tubulin antibody. The second row (the channel showing tubulin) shows the sum of the total series of sections of the cell, showing the presence of both kinetochore and non-kinetochore microtubules in the spindle. (B) A cell irradiated with a single line in one focal plane: microtubules remained in other layers (shown by three images from up and down focal planes). (C) A cell irradiated with a single line in multiple focal planes and fixed after the irradiated pole started to move toward the equator: microtubules were completely absent in the irradiated region in all focal planes (shown by three images in widely separate focal planes). (D) A cell irradiated with a line in multiple focal planes and fixed immediately after irradiation, before the irradiated pole started to move toward the equator: all microtubules disappeared in the irradiated site (shown by three images in widely separate focal planes). Bar: $5 \mu \mathrm{m}$.

Figure 5 (A) The effect of different actin and myosin inhibitors on the spindle lengths of PtK2 cells before irradiation. (B and C) The consequences of line cuts in spindles of different lengths in cells treated with different (B) actin and (C) myosin inhibitors before laser microbeam irradiation. (D and E) The effect of different actin and myosin inhibitors on the shortening percentage of the entire spindle length upon laser microbeam irradiation. These data are only for those cells in which at least one pole moved: all other cells were omitted. (F and G) The effect of different actin and myosin inhibitors on the inward movement velocities of the poles on the irradiated and un-irradiated sides of the spindle. For both sets of measurements only those poles that moved were included. The measurements obtained for the spindle lengths (on the X axis) in 
each bin were from the lower value up to and smaller than the higher value. Thus for values indicated as 6 to 8 , the values were 6 to $<8$, and those indicated as 8 to 10 were 8 to $<10$, and so forth. (We assessed differences between average values using student's t-test.: *: $\mathrm{P} \leq 0.05$; **: $\mathrm{P}$ $\leq 0.01$. The error bars indicate the standard error of the mean.)

Figure 6 (A) PtK2 cells stained with tubulin antibody and Phalloidin. First row: control (untreated) cell; second row: CD treated cell; third row: LatB treated cell. The arrow points to a buckled microtubule in a LatB-treated cell. Bar: $5 \mu \mathrm{m}$. (B) Graph of distance from the equator (in $\mu \mathrm{m}$ ) of the poles on the irradiated and un-irradiated sides, versus time in minutes, for $2 \mathrm{LatB}$ treated cells, illustrating the effects of an actin inhibitor. The (blue) crosses represent the positions of the pole on the irradiated side of the spindle and the (red) triangles represent the positions of the other pole. The vertical line represents the time of the irradiation. In the one cell both poles move very slightly, less than $1 \mu \mathrm{m}$, toward the equator. In the other cell only the pole on the not-irradiated side moves toward the equator.

Figure 7 (A) PtK2 cells stained with tubulin antibody and MY-21 antibody to stain total myosin, as indicated: the top row is a control cell and the bottom row a BDM treated cell. The arrow points to a bent microtubule bent in the BDM treated cell. Bar: $5 \mu \mathrm{m}$. From the images in the myosin channel it might appear that the chromosomes are stained, but as discussed in the text this is an inconsistently arising artefact of our procedure (see Fig 7C) that we have not been able to eliminate. (B) PtK2 cells stained with tubulin antibody and 3675 Cell Signaling antibody to stain 1P-myosin: First row: control (un-treated) cell; second row: Y-27632 treated cell; third row: ML-7 treated cell. The arrowheads point to the absence of asters in the cells treated with Y27532 and ML-7. The arrow points to a bent microtubule in the ML-7 treated cell. Bar: $5 \mu \mathrm{m}$. (C) Confocal microscope images of a PtK2 cell not stained with any antibody, showing that the chromosomes appear stained but there is no spindle staining, and thus that the weak staining seen with myosin antibodies in 7A and 7B represents actual staining. Bar: $5 \mu \mathrm{m}$. (D and E) Graphs of distance of the irradiated and un-irradiated poles from the equator in $\mu \mathrm{m}$, versus time in minutes, for 2 cells treated with Y-27632. The (blue) crosses represent the positions of the pole on the irradiated side of the spindle and the (red) triangles represent the positions of the other pole. The 
vertical line represents the time of the irradiation. (D) represents a cell in which neither pole moved to the equator. (E) represents a cell in which only the pole on the not-irradiated side moved toward the equator.

Figure 8 (A) PtK2 cell irradiated in one focal plane with a single line (in the top left halfspindle) and a rectangle (in the bottom right half-spindle) and stained for tubulin and actin: the magnified section in merged picture illustrates the presence of actin in the irradiated region seemingly adjacent to microtubules that remain in the un-irradiated region. Red and green arrows point to remaining actin and microtubule in the irradiated region. (B) PtK2 irradiated with a line in multiple focal planes and stained for tubulin and 1P-myosin: magnified section in merged picture indicates the presence of 1P-myosin and the absence of microtubules in the irradiated region. Red arrowheads point to 1P-myosin localisation in the irradiated region. Bar: $5 \mu \mathrm{m}$.

Figure 9 Models representing possible interactions between microtubules, actin, myosin and spindle matrix: (A) Illustrates myosin linked to actin by its head domain and to microtubules by its tail domain. (B) Illustrates myosin linked to the spindle matrix by its tail and to actin by its head, and that actin filaments and microtubules are linked (vertical blue lines).

\section{Supplementary movie legends:}

Movie 1 Time lapse imaging of the PtK2 cell illustrated in figure 1A. The first image after laser irradiation was recorded at 10:38:12. The spindle fibre on the un-irradiated site bent as the pole moved towards the equator. The sequence covers a period of $7 \mathrm{~min}$ and $44 \mathrm{~s}$. 
Table 1 Summary of the laser microbeam irradiation on the irradiated and un-irradiated half-spindle lengths and equatorial movement velocities, average values $+/-$ standard errors in control cells irradiated in one focal plane (A) and in multiple focal planes (B). (C) The effect of laser microbeam irradiation on equatorial movement of irradiated and un-irradiated poles in control, CD, LatB, JASP, BDM, Y-27632 and ML-7 treated cells.

A

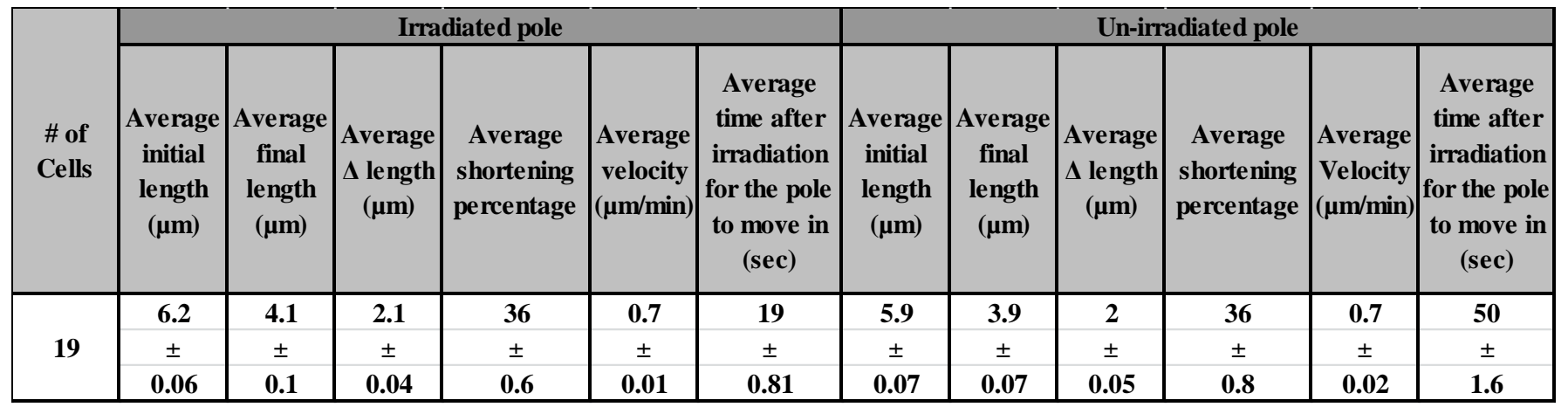

B

\begin{tabular}{|c|c|c|c|c|c|c|c|c|c|c|c|c|}
\hline \multirow[b]{2}{*}{$\begin{array}{l}\text { \# of } \\
\text { Cells }\end{array}$} & \multicolumn{6}{|c|}{ Irradiated pole } & \multicolumn{6}{|c|}{ Un-irradiated pole } \\
\hline & $\begin{array}{c}\text { Average } \\
\text { initial } \\
\text { length } \\
(\mu \mathrm{m})\end{array}$ & $\begin{array}{c}\text { Average } \\
\text { final } \\
\text { length } \\
(\mu \mathrm{m})\end{array}$ & $\begin{array}{c}\text { Average } \\
\Delta \text { length } \\
(\mu \mathrm{m})\end{array}$ & $\begin{array}{c}\text { Average } \\
\text { shortening } \\
\text { percentage }\end{array}$ & $\begin{array}{c}\text { Average } \\
\text { velocity } \\
(\mu \mathrm{m} / \mathrm{min})\end{array}$ & $\begin{array}{c}\text { Average } \\
\text { time after } \\
\text { irradiation } \\
\text { for the pole } \\
\text { to move in } \\
\text { (sec) }\end{array}$ & $\begin{array}{c}\text { Average } \\
\text { initial } \\
\text { length } \\
(\mu \mathrm{m})\end{array}$ & $\begin{array}{c}\text { Average } \\
\text { final } \\
\text { length } \\
(\mu \mathrm{m})\end{array}$ & $\begin{array}{c}\text { Average } \\
\Delta \text { length } \\
(\mu \mathrm{m})\end{array}$ & $\begin{array}{c}\text { Average } \\
\text { shortening } \\
\text { percentage }\end{array}$ & $\begin{array}{l}\text { Average } \\
\text { Velocity } \\
(\mu \mathrm{m} / \mathrm{min})\end{array}$ & $\begin{array}{c}\text { Average } \\
\text { time after } \\
\text { irradiation } \\
\text { for the pole } \\
\text { to move in } \\
\text { (sec) }\end{array}$ \\
\hline \multirow{3}{*}{4} & 8.7 & 6.4 & 2.3 & 23.6 & 1.1 & 10.7 & 7.7 & 5.6 & 2.2 & 29.3 & 0.8 & 32 \\
\hline & \pm & \pm & \pm & \pm & \pm & \pm & \pm & \pm & \pm & \pm & \pm & \pm \\
\hline & 0.6 & 0.4 & 0.7 & 7.6 & 0.03 & 0.6 & 0.7 & 0.8 & 0.4 & 5.8 & 0.2 & 4.3 \\
\hline
\end{tabular}

C

\begin{tabular}{|c|c|c|c|c|c|}
\hline Treatment & $\begin{array}{c}\text { Total } \\
\text { number } \\
\text { of cells }\end{array}$ & $\begin{array}{c}\text { Number of cells } \\
\text { with movement of } \\
\text { irradiated and } \\
\text { unirradiated poles }\end{array}$ & $\begin{array}{c}\text { Number of cells } \\
\text { with movement } \\
\text { of irradiated } \\
\text { pole only }\end{array}$ & $\begin{array}{c}\text { Number of cells } \\
\text { with movement } \\
\text { of unirradiated } \\
\text { pole only }\end{array}$ & $\begin{array}{c}\text { Number of cells } \\
\text { with no movement } \\
\text { of the poles }\end{array}$ \\
\hline Control, irradiated in one focal plane & 19 & 14 & 2 & 3 & 0 \\
\hline Control, irradiated in multiple focal planes & 4 & 3 & 1 & 0 & 0 \\
\hline CD & 8 & 2 & 1 & 1 & 4 \\
\hline LatB & 7 & 2 & 1 & 2 & 2 \\
\hline JASP & 9 & 1 & 3 & 1 & 4 \\
\hline BDM & 7 & 2 & 2 & 1 & 2 \\
\hline Y-27632 & 8 & 1 & 1 & 3 & 3 \\
\hline ML-7 & 16 & 1 & 2 & 3 & 10 \\
\hline
\end{tabular}




\section{A. Single line irradiation, one focal plane}
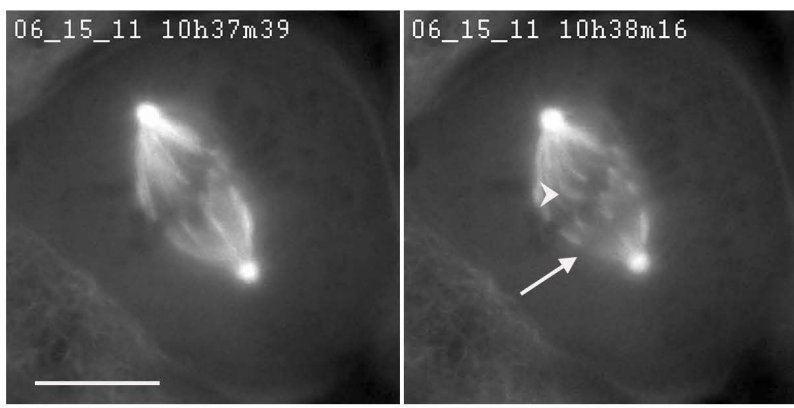

06_15_11 $10 \mathrm{~h} 38 \mathrm{~m} 20$

06_15_11 10h38m28

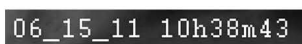

06_15_11 10h39m10

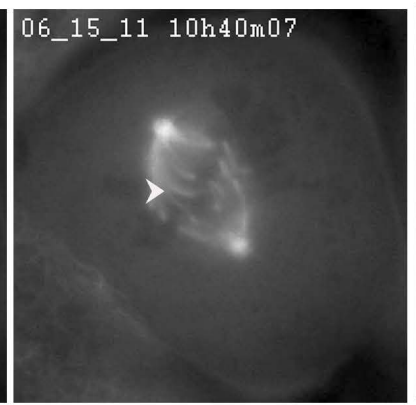

06_15_11 10h38m33
06_15_11 10h38m25

06_15_11 10h38m36

$06 \_15 \_11$ 10h38m40

$06 \_15 \_11 \quad 10 \mathrm{~h} 38 \mathrm{~m} 56$

$06 \_15 \_11 \quad 10 \mathrm{~h} 38 \mathrm{~m} 52$

06_15_11 10h41m09

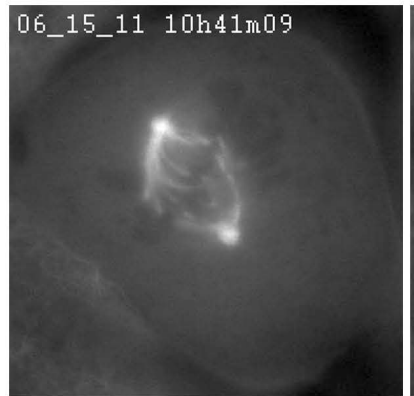

06_15_11 10h42m04

\section{6_15_11 $10 \mathrm{~h} 38 \mathrm{n} 56$}
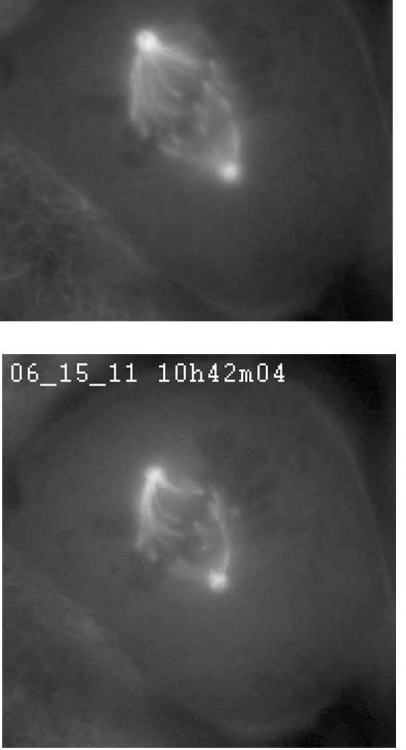

Figure 1A 
B: Single line irradiation, multiple focal planes
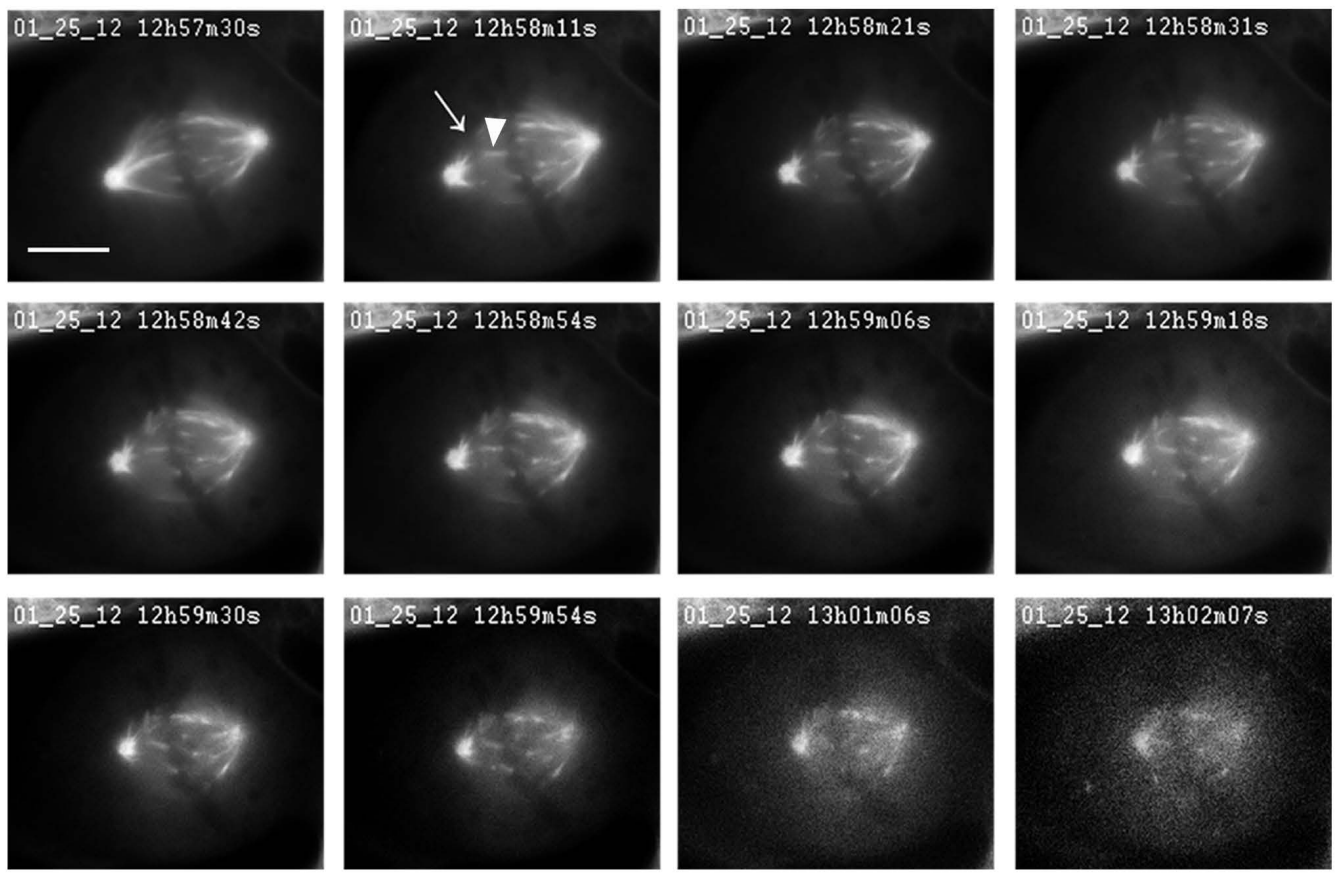

Figure 1B 
A : Single line irradiation, one focal plane

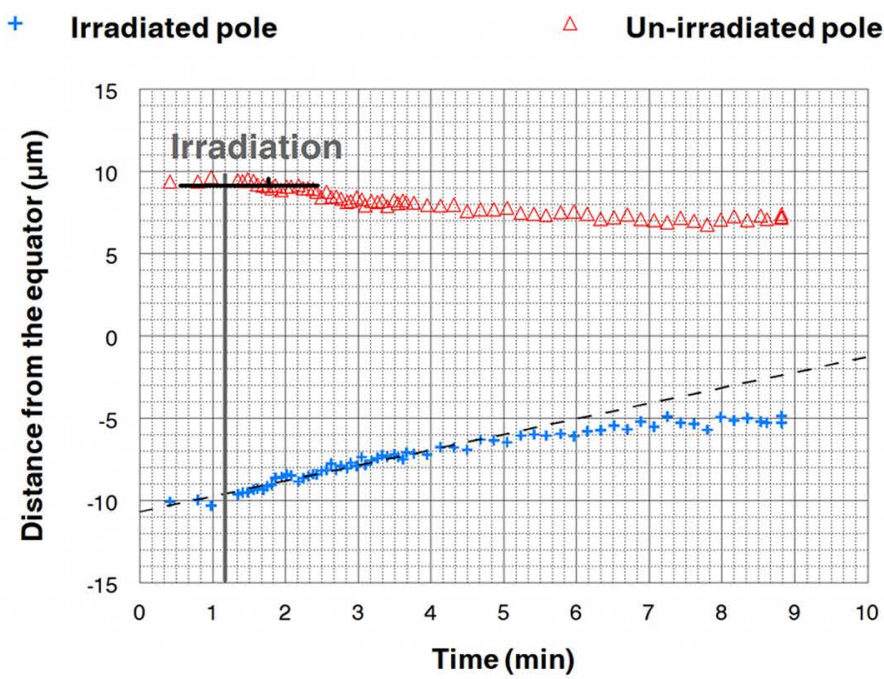

B: Single line irradiation, multiple focal planes

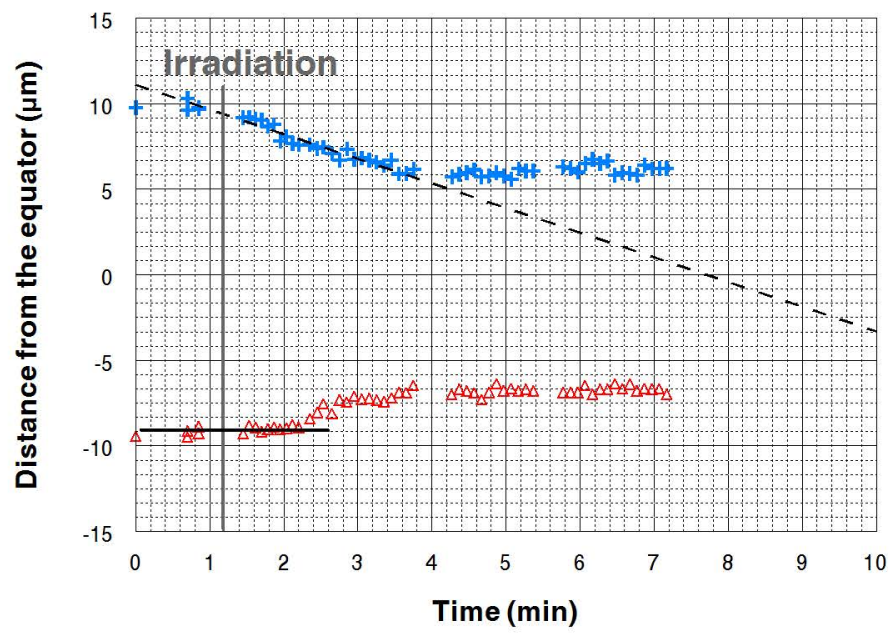

C: Repeated irradiations, one focal plane

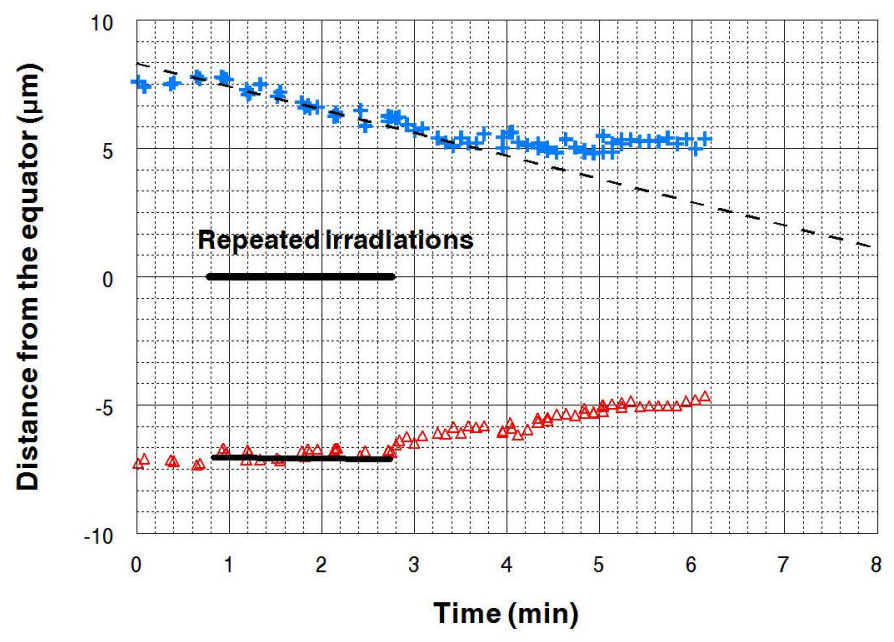

Figure 2 
A

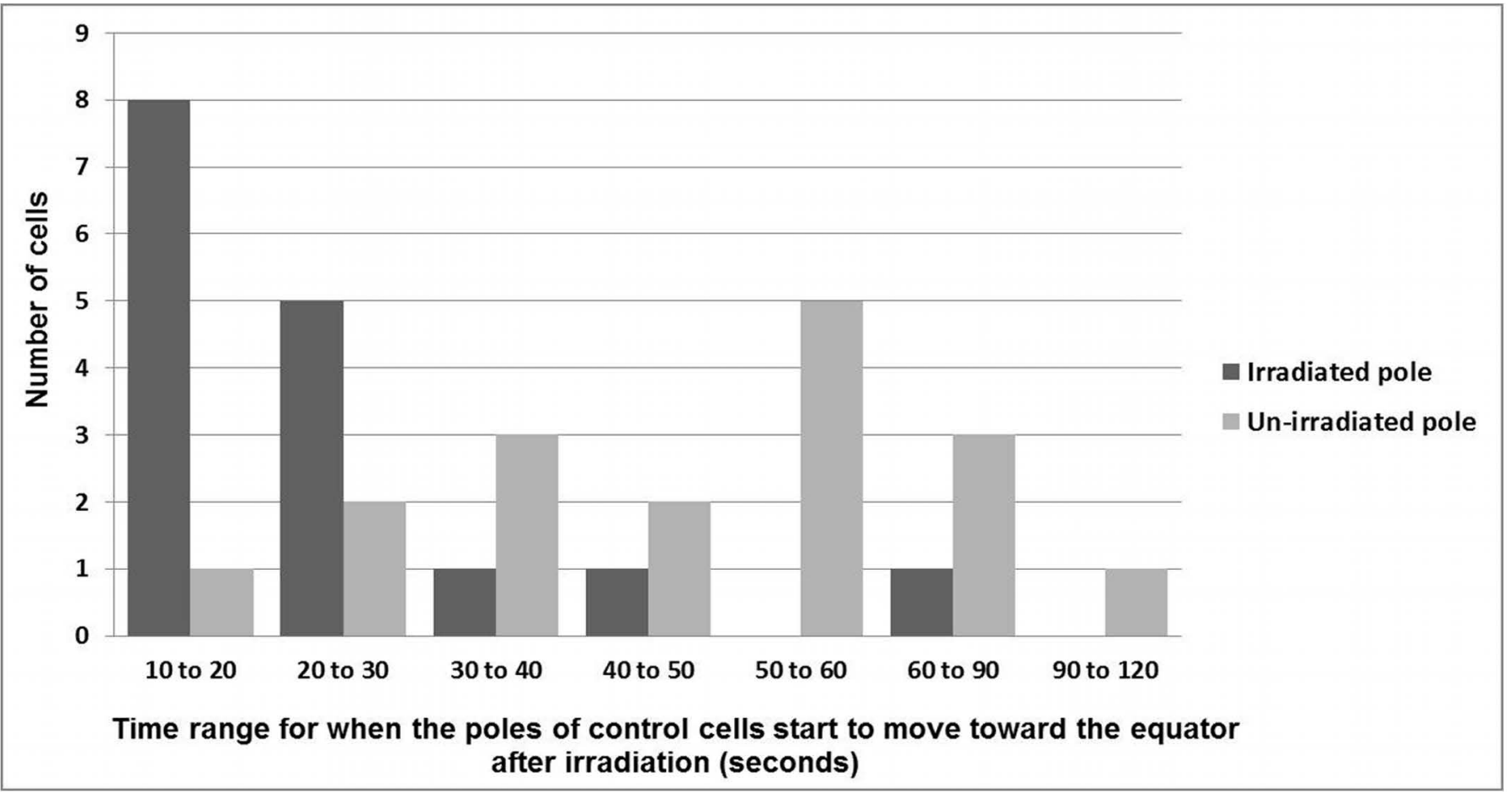

B

$\boldsymbol{D B o t h}$ poles moved in $\boldsymbol{\square}$ One pole moved in

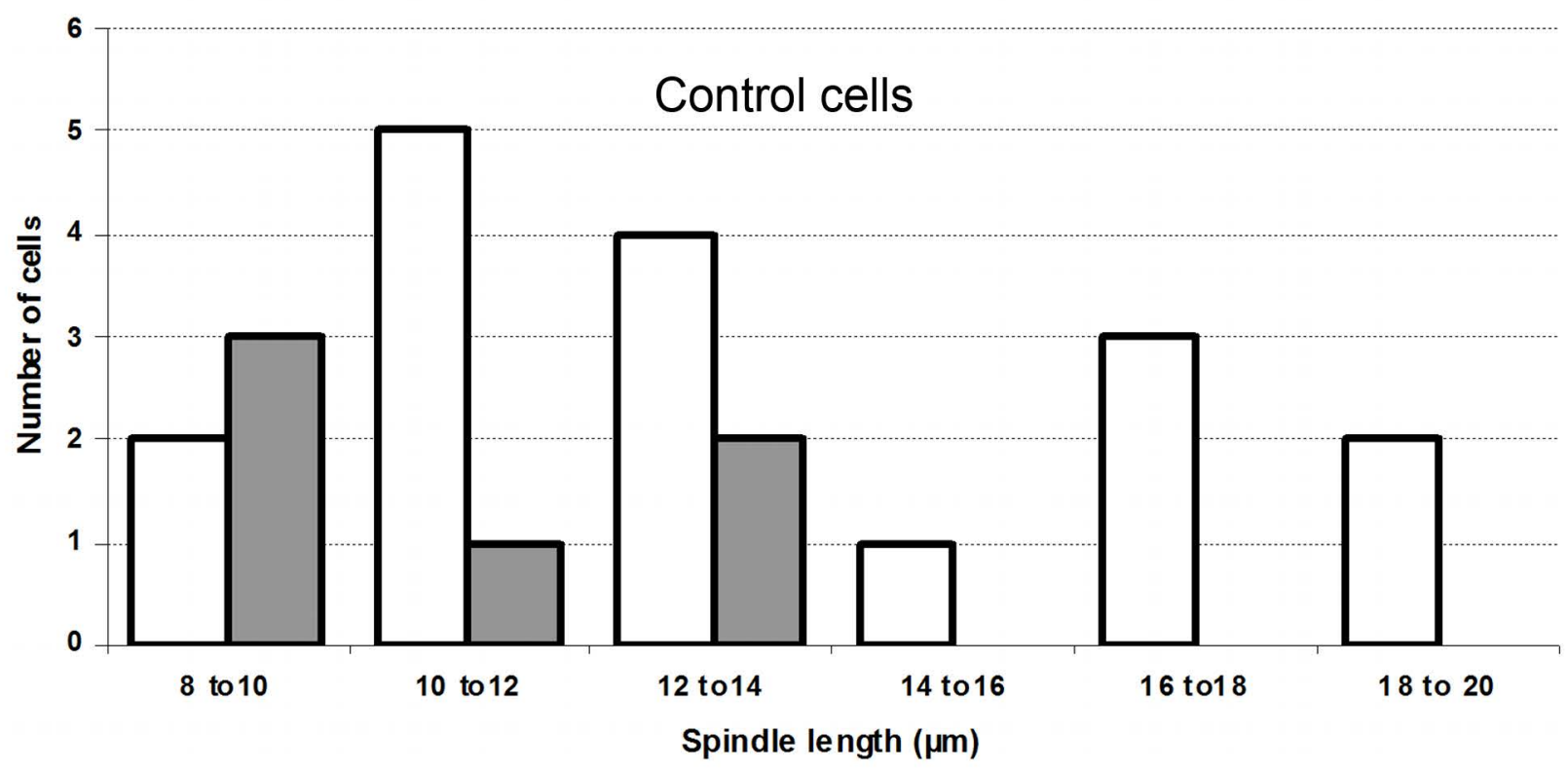

Figure 3 

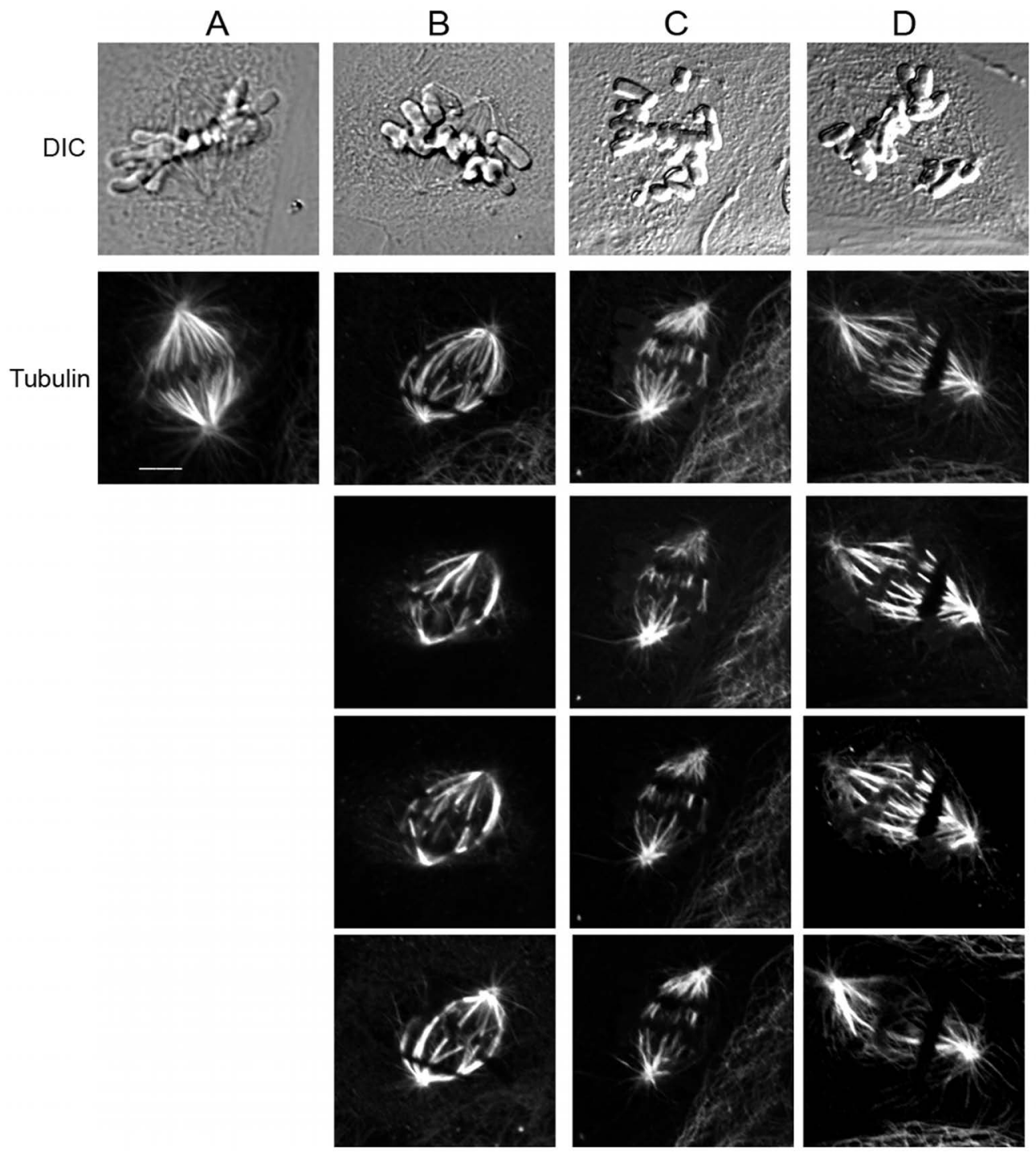

Figure 4 
A

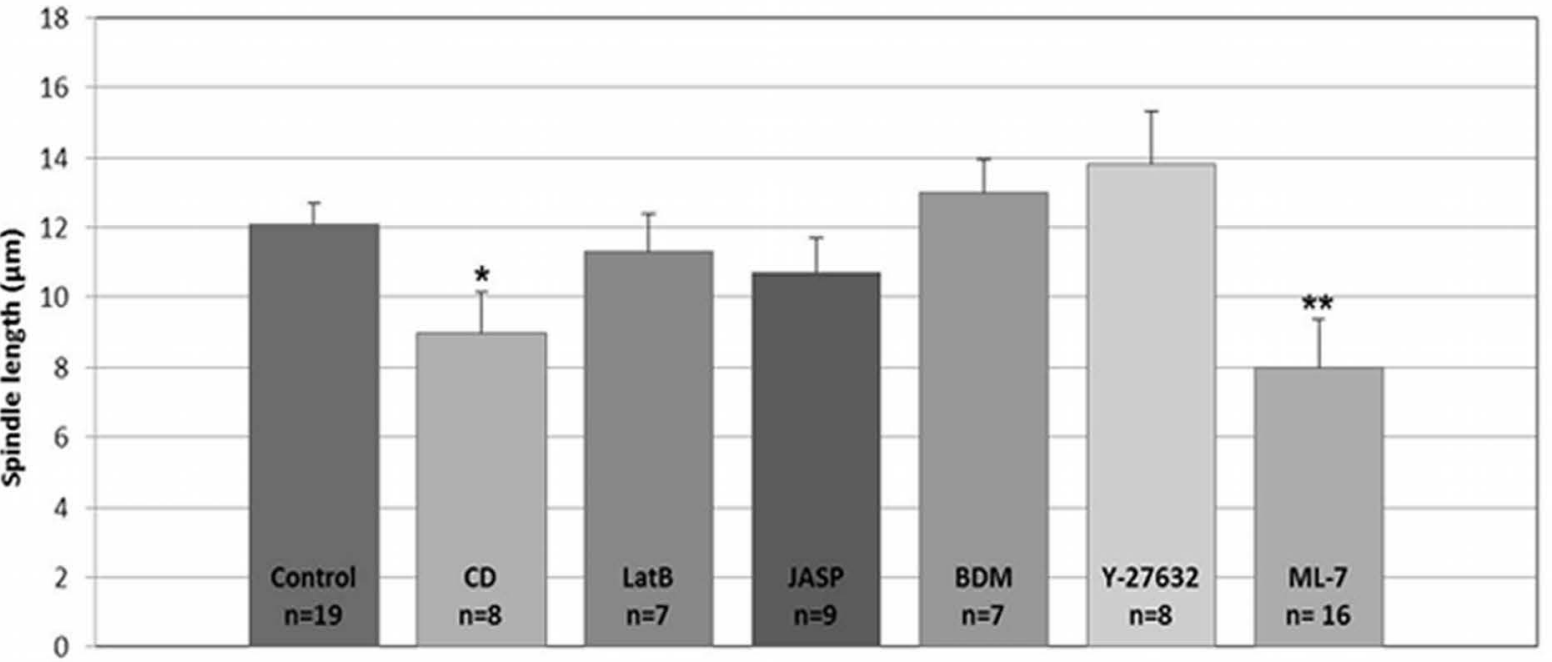

B $\quad$ Both poles moved in $\square$ One pole moved in $\square$ Neither pole moved in

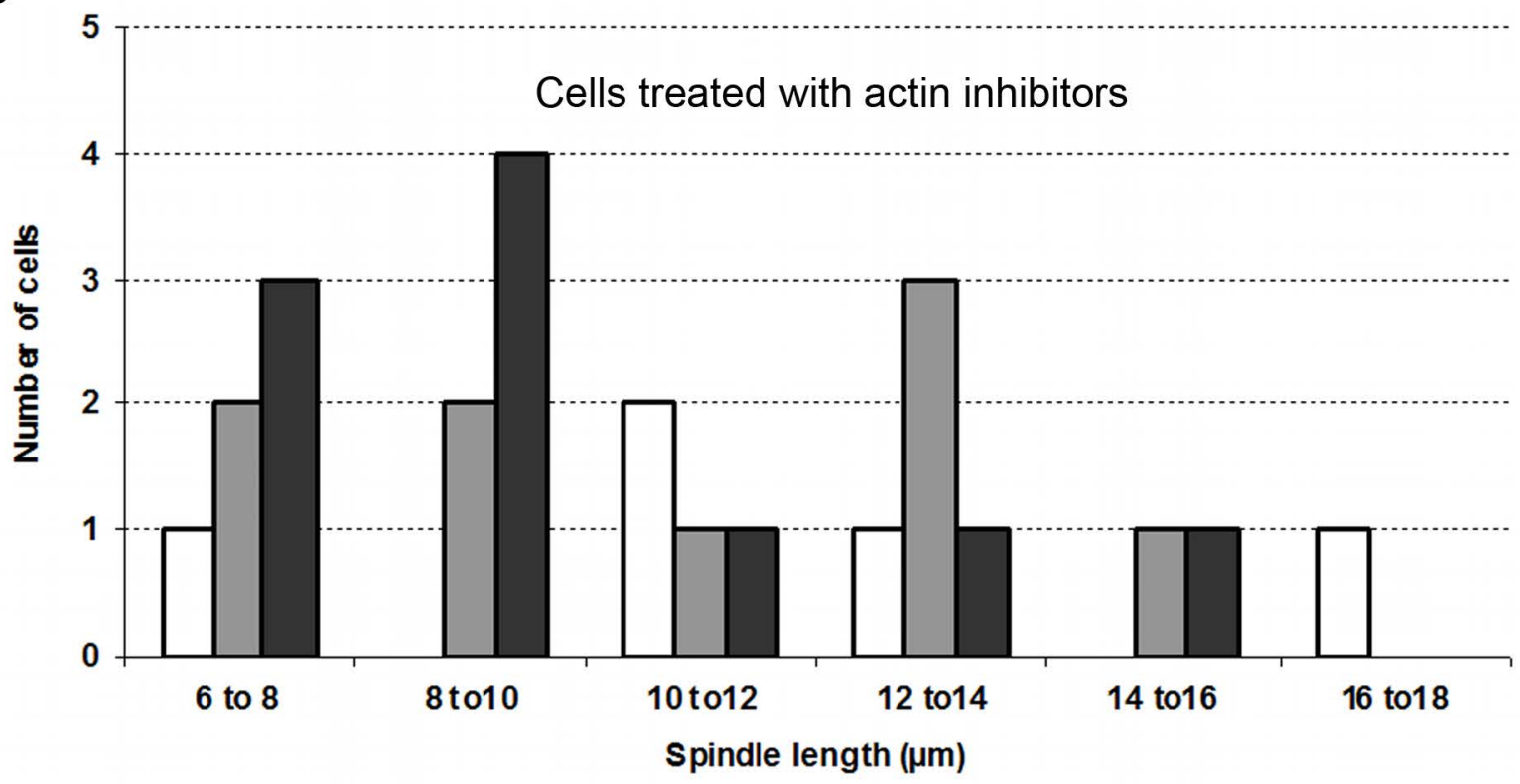

C $\quad$ Both poles moved in $\mathbf{D}$ One pole moved in $\mathbf{a}$ Neither pole moved in

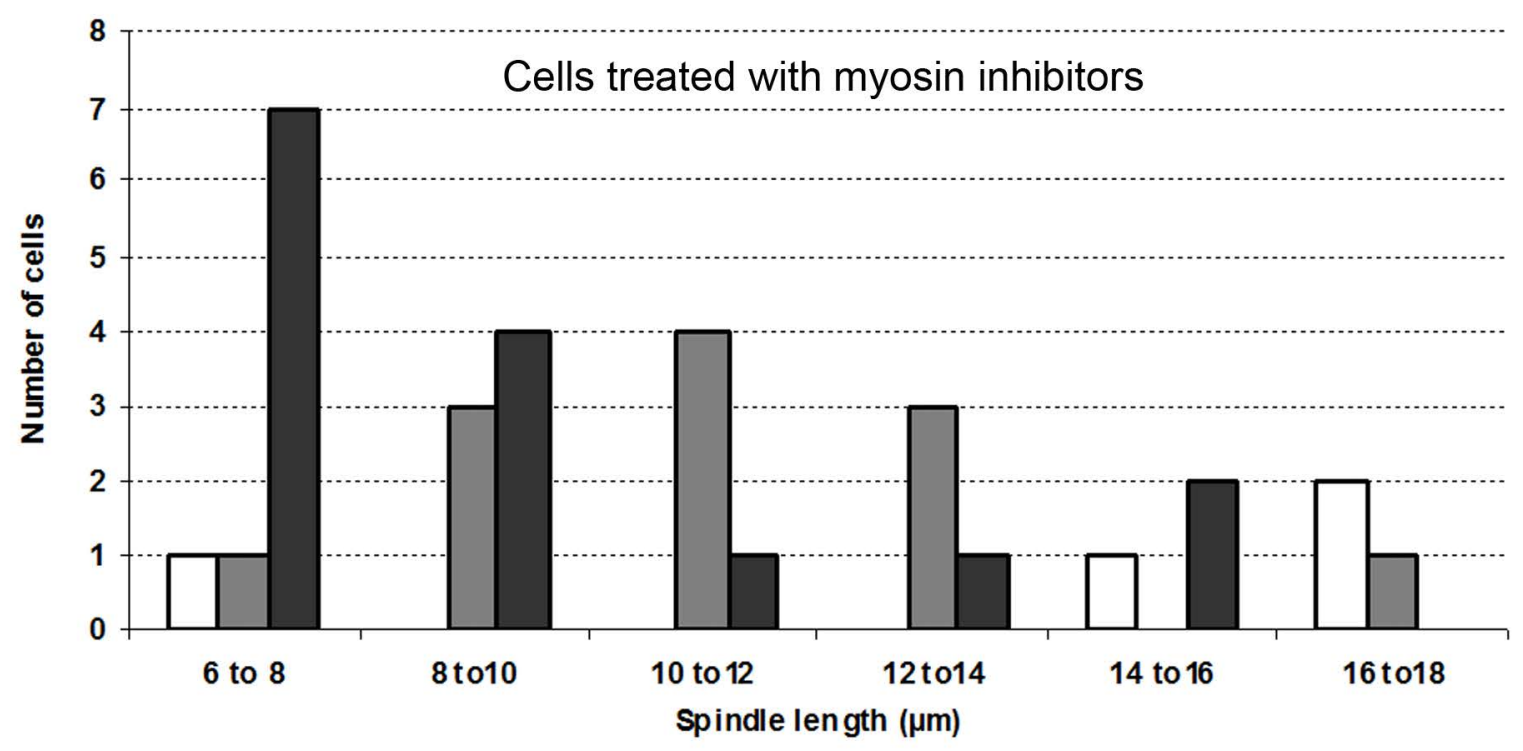


D

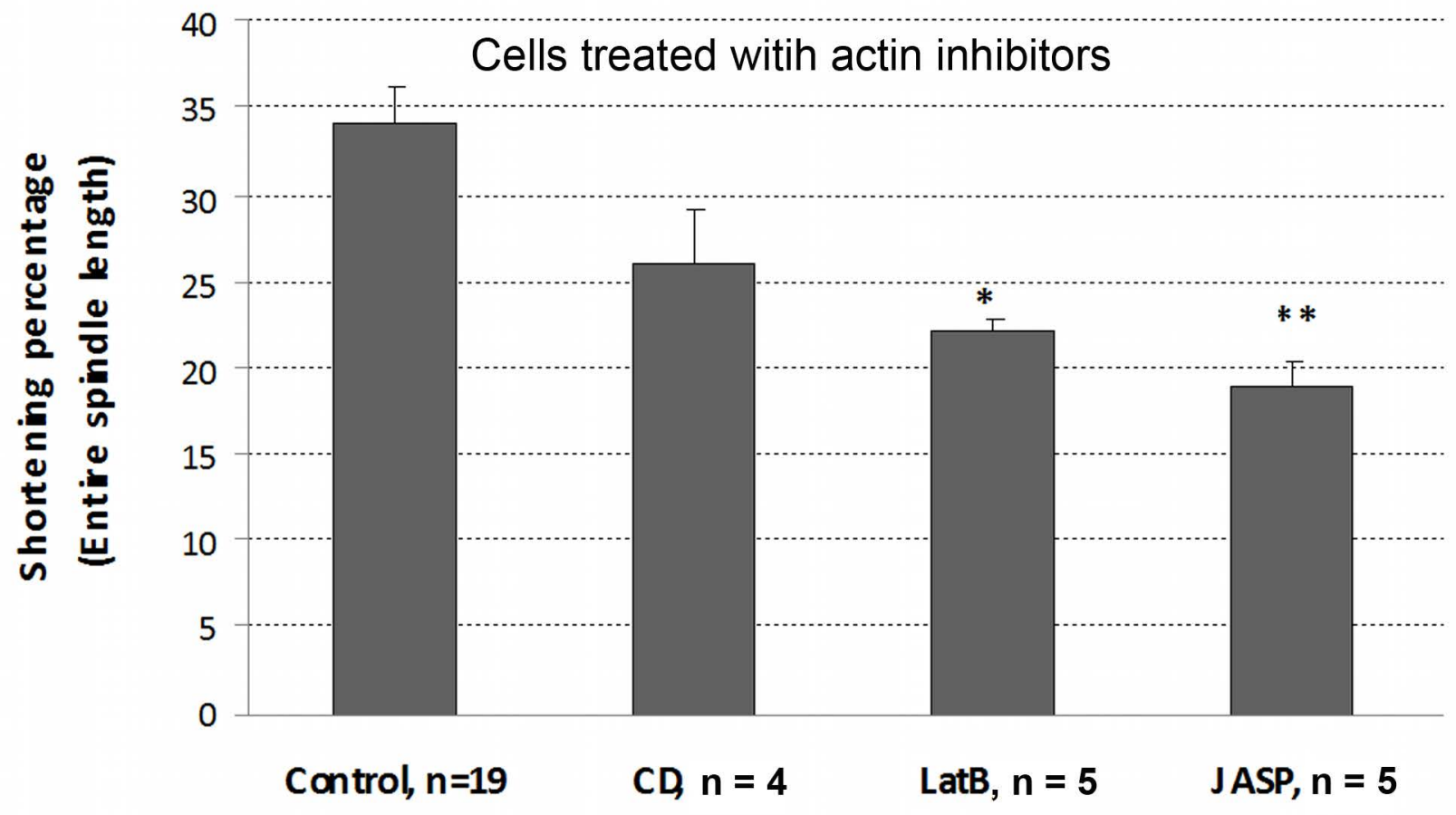

E

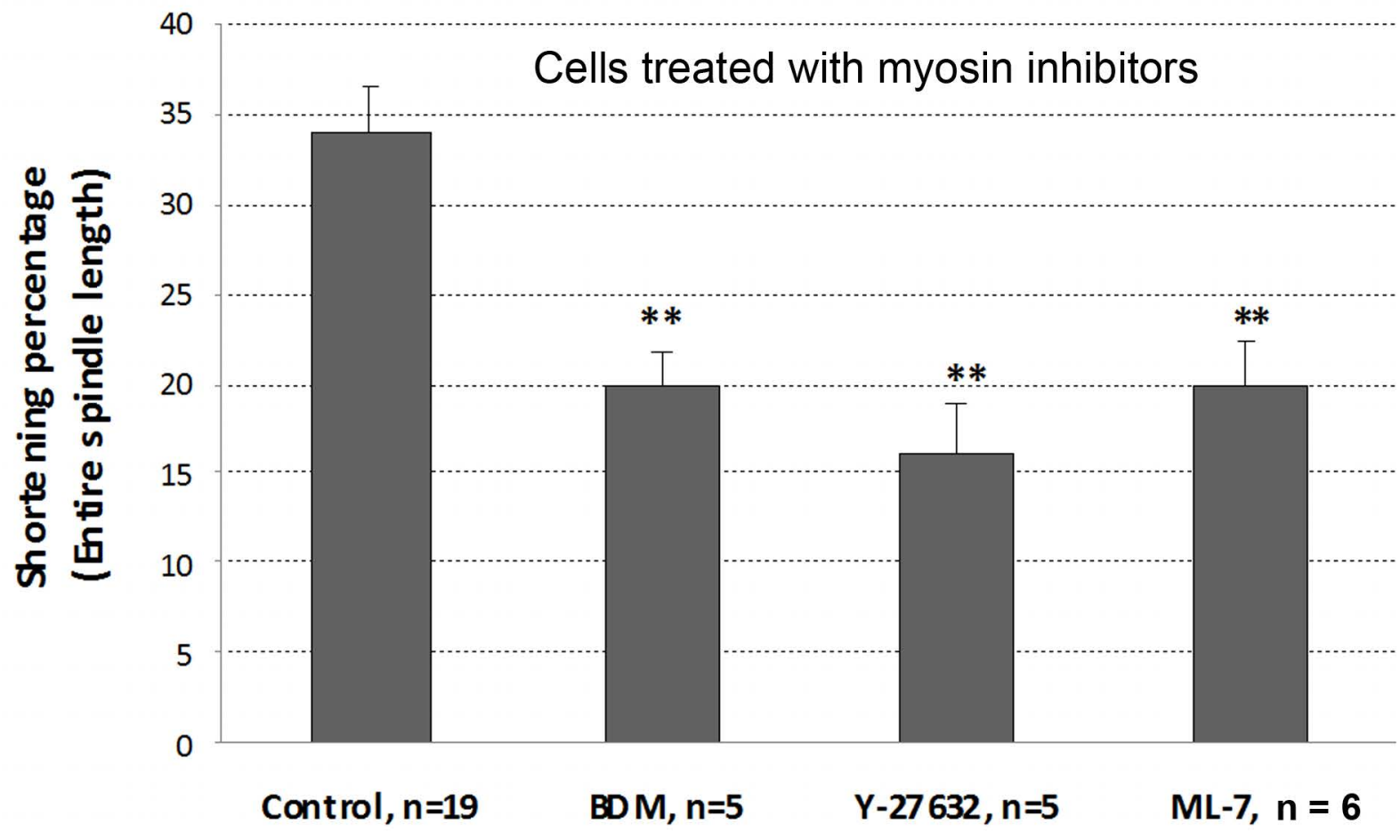

Figure 5 


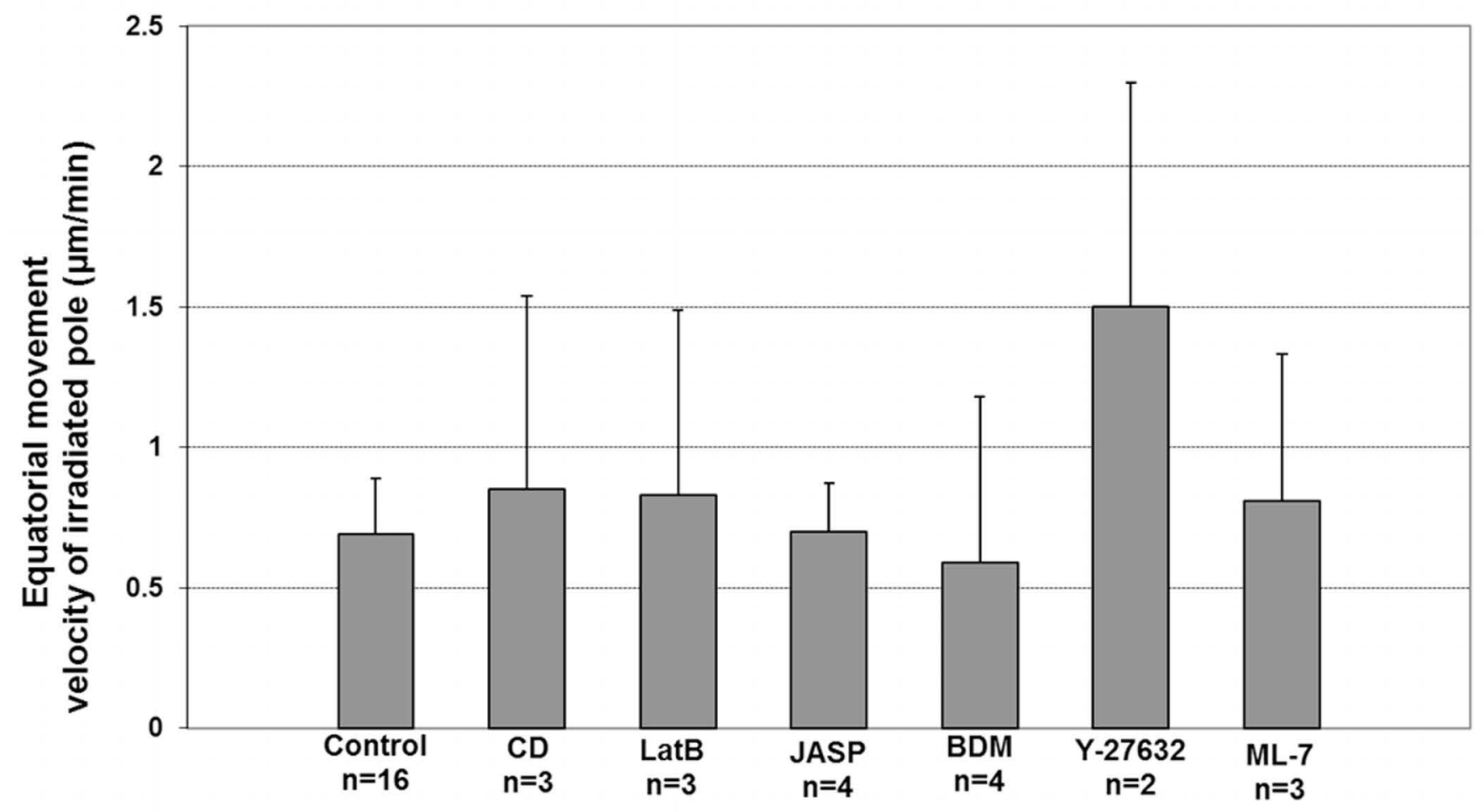

G

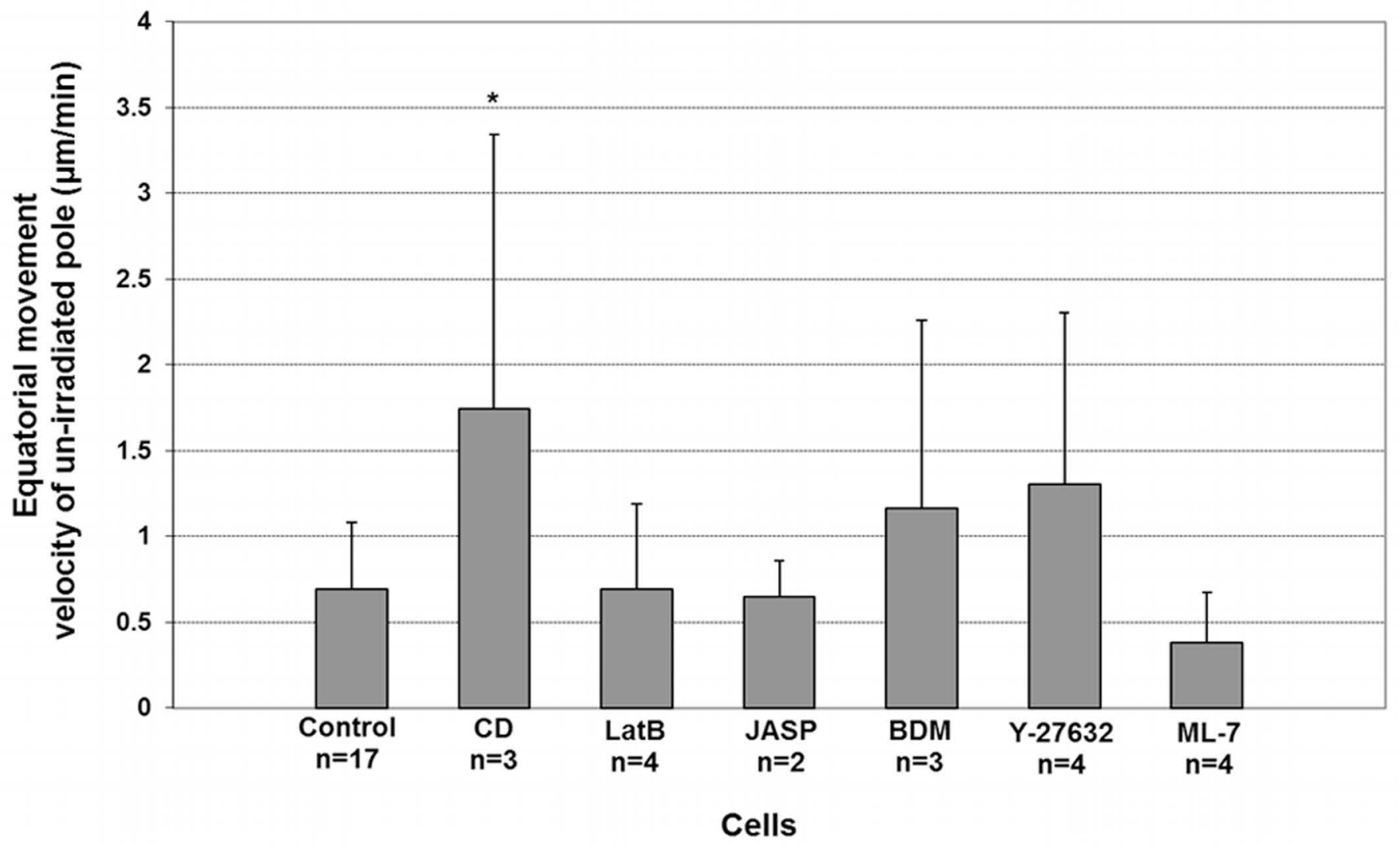

Figure 5 
A

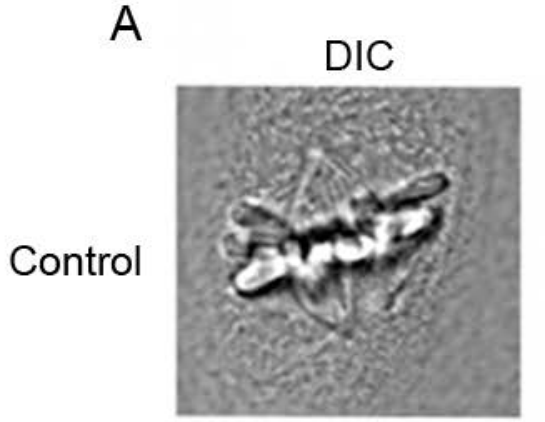

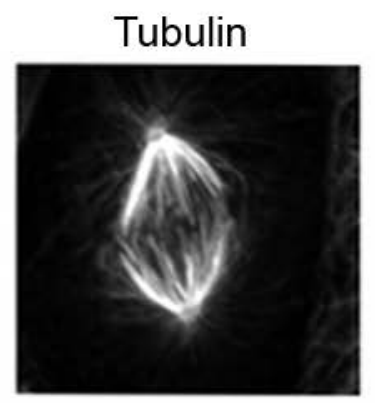
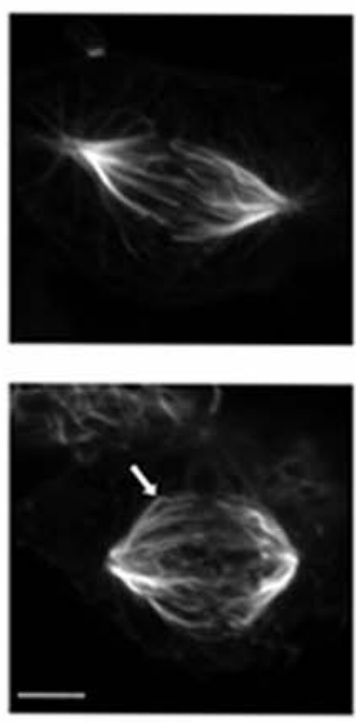

Spindle actin
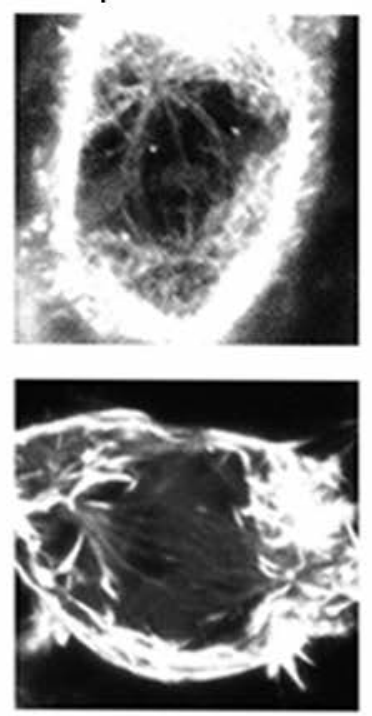

Cortical actin
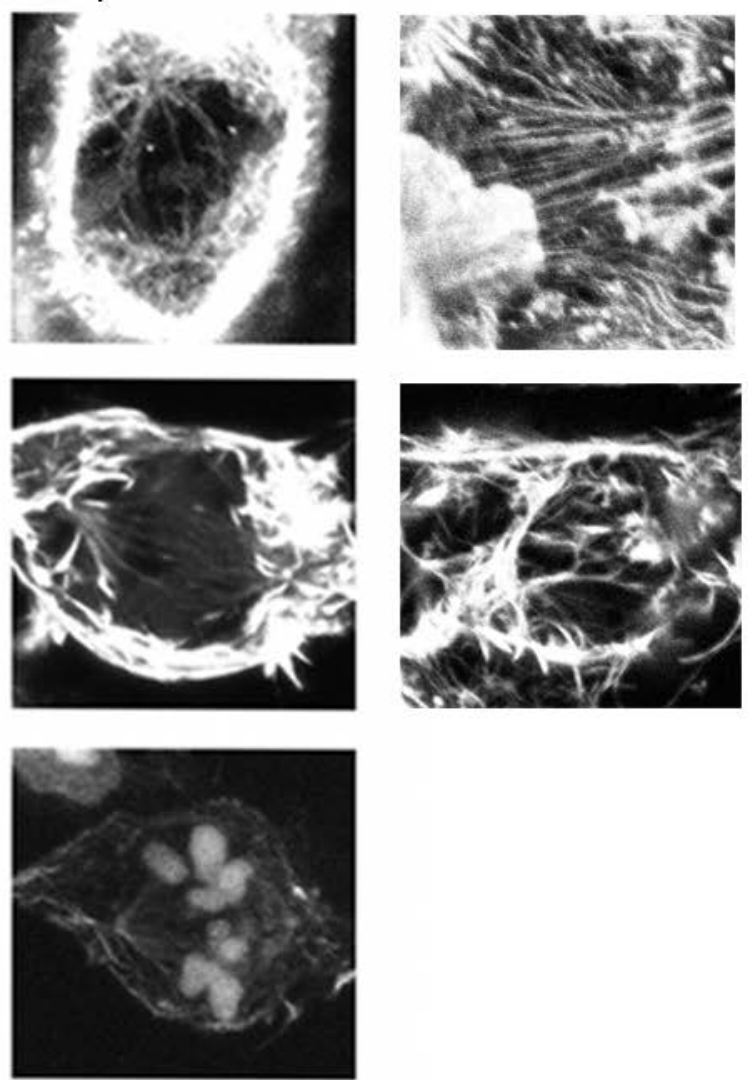

B

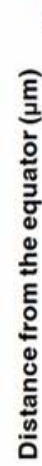

$+\quad$ Irradiated pole

LatB treated cell
Un-irradiated pole

15 Irradiation

10

.$$
\text { -10 }
$$

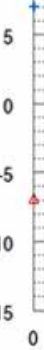

.

1
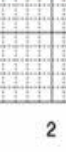

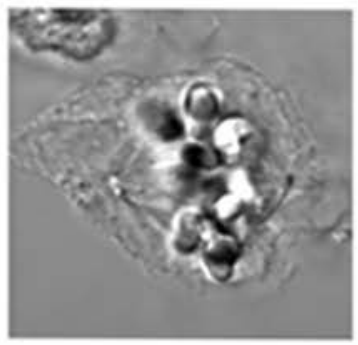

LatB treated cell

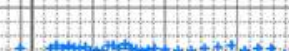

$++t+t+t$

$+t+t+t+t+t+t+t+$
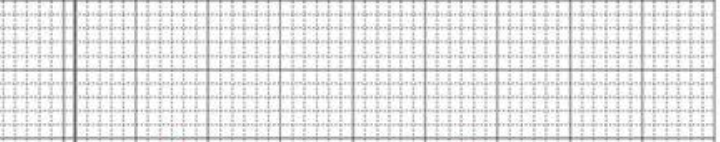

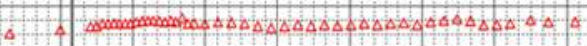

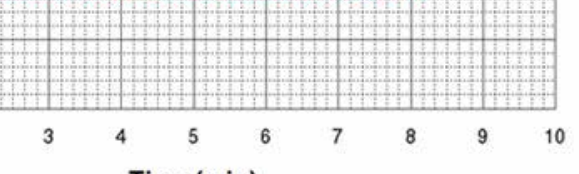

Time (min)

C

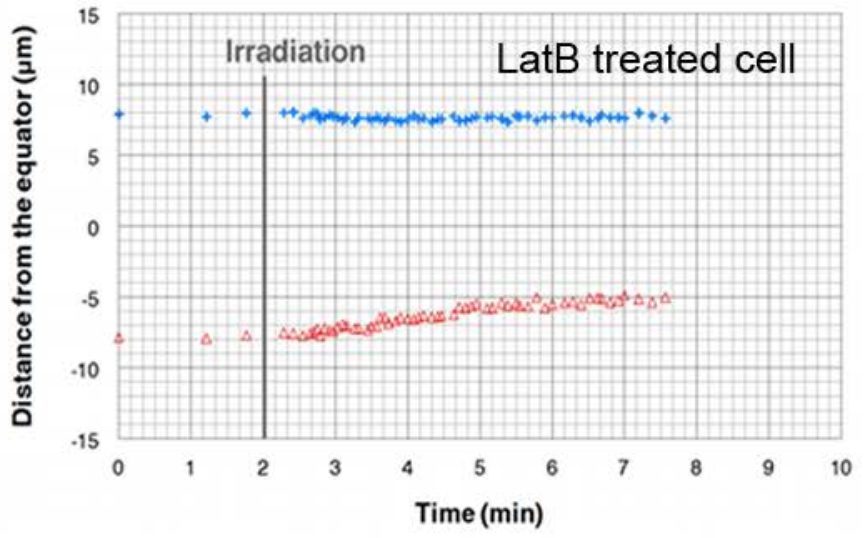


A
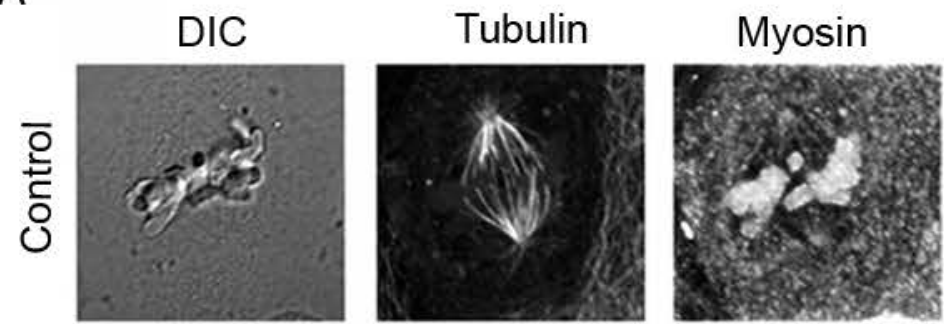

C
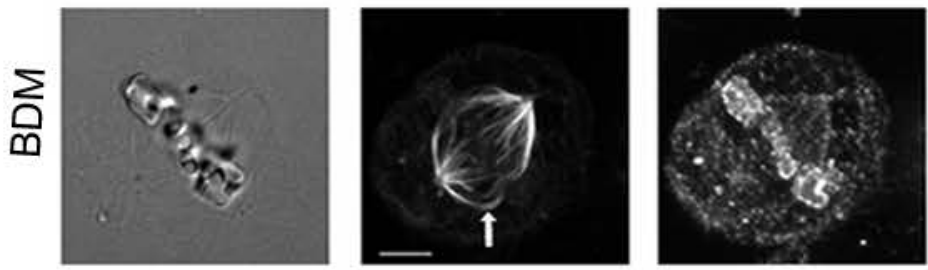

B

DIC
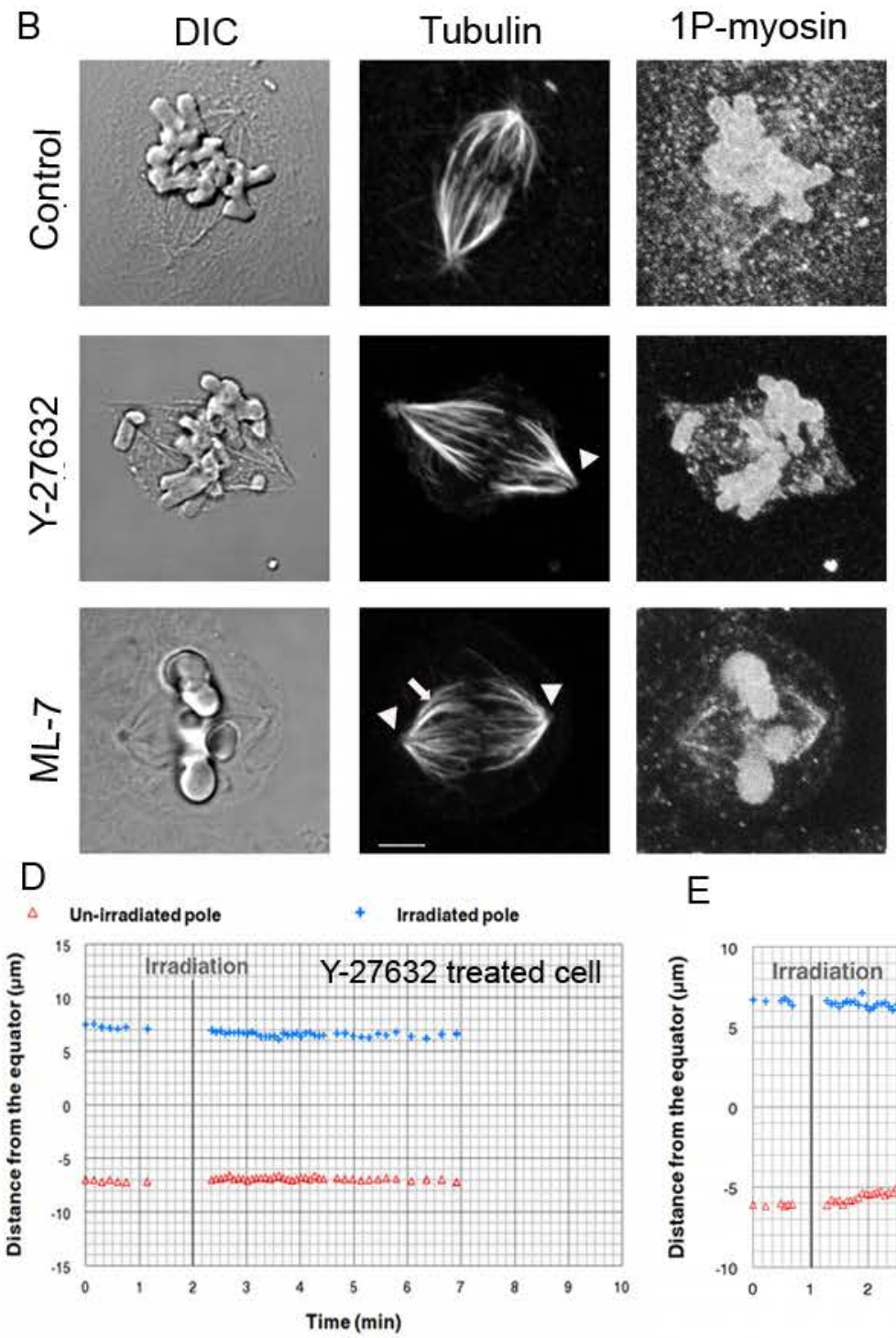

E
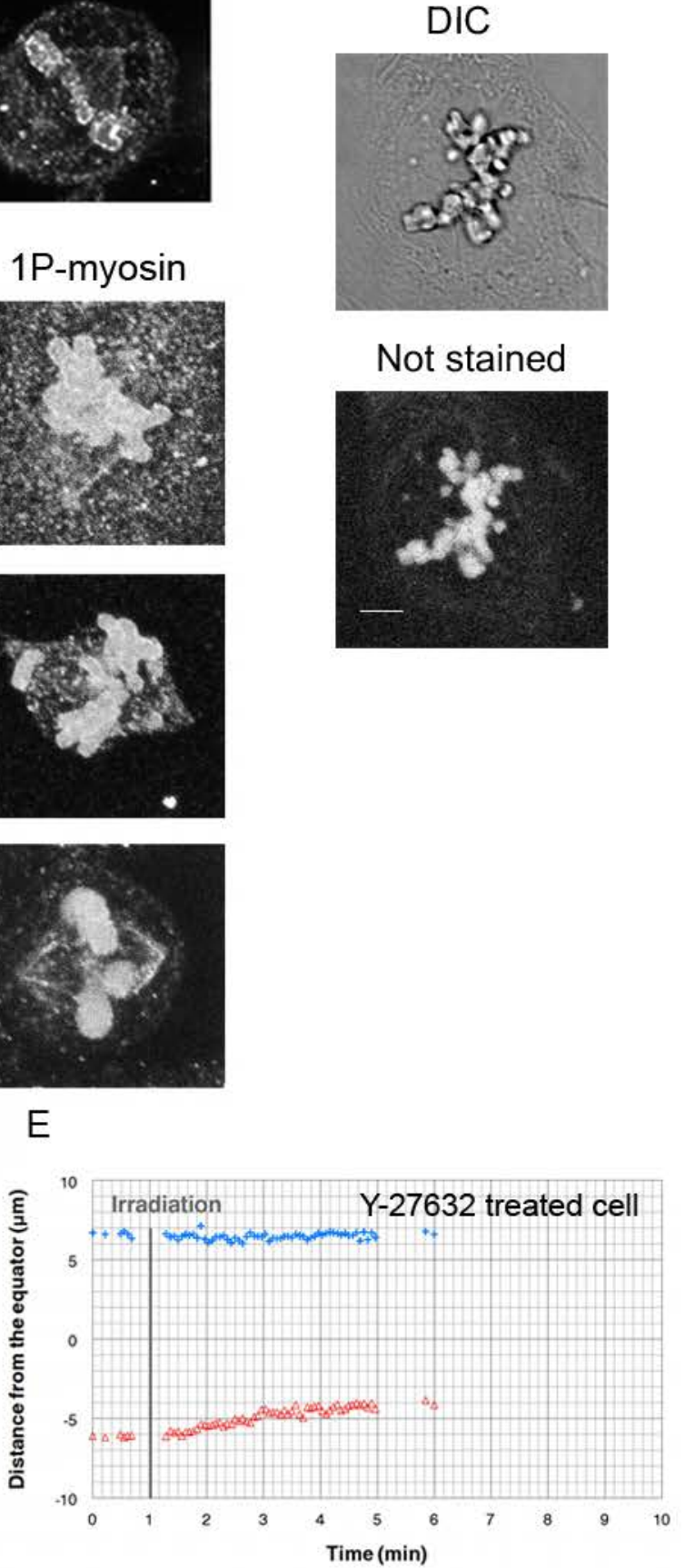

Figure 7 
A

Tubulin

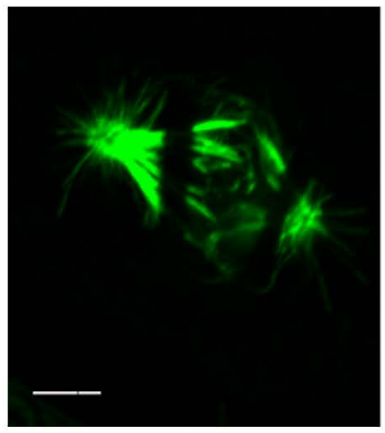

B

Tubulin

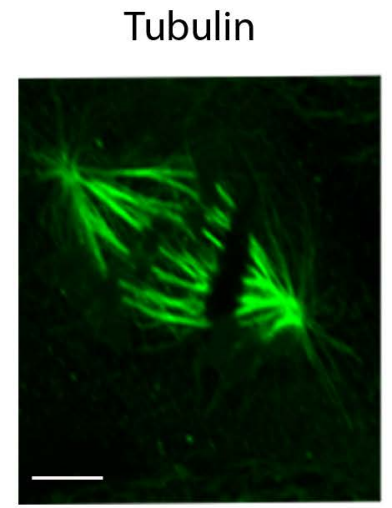

Actin

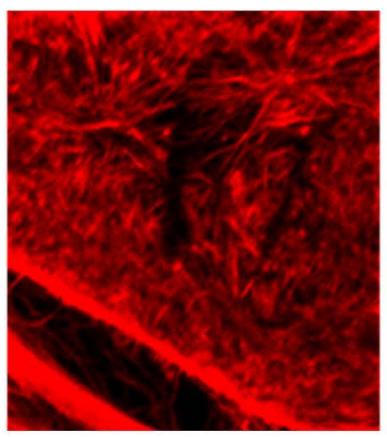

1P-myosin

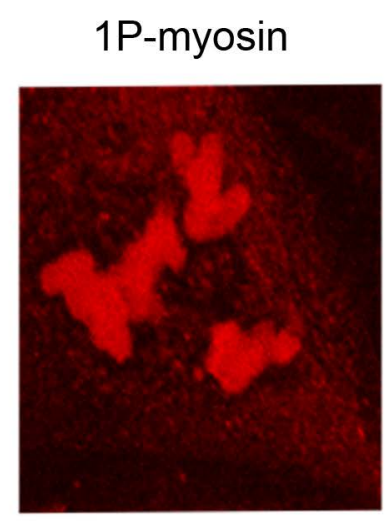

Merged

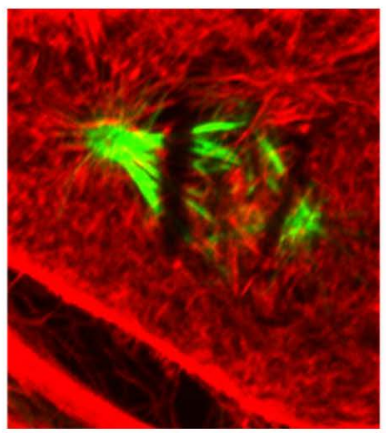

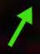

Merged

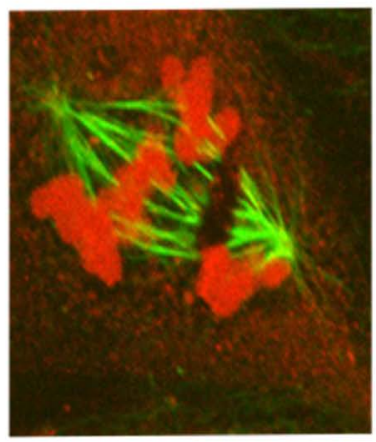

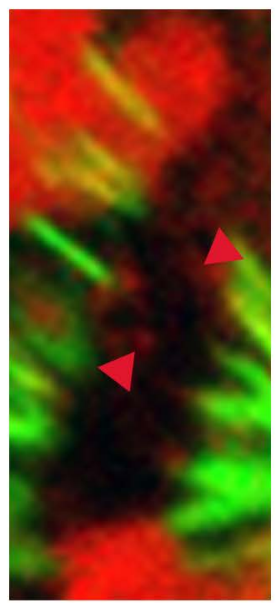

Figure 8 
A

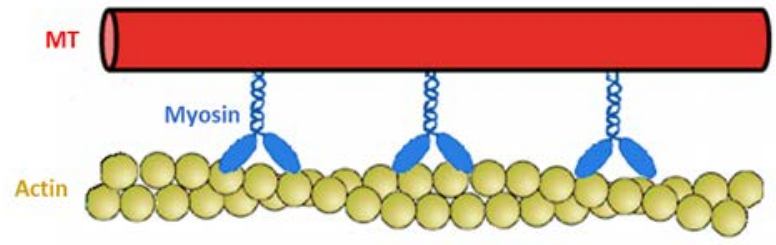

B

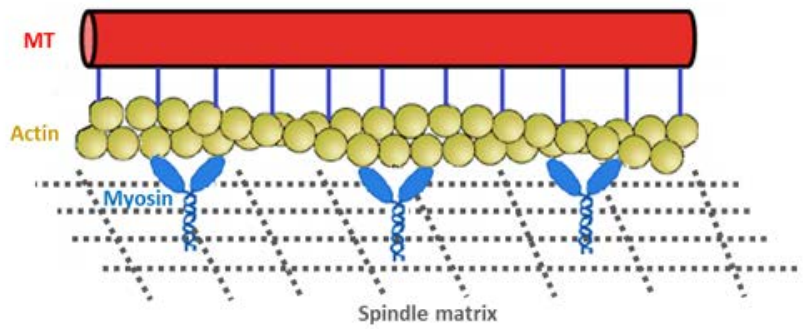

Figure 9 